\title{
Guidance of attention by working memory is a matter of representational fidelity
}

\author{
Jamal R. Williams ${ }^{1}$, Timothy F. Brady ${ }^{1}$, \& Viola S. Störmer ${ }^{1,2}$
}

1. Department of Psychology, University of California, San Diego

2. Department of Psychological and Brain Sciences, Dartmouth College

Word Count: 14960

Please address correspondence to:

Jamal R. Williams

Department of Psychology

University of California, San Diego

9500 Gilman Drive \#0109

La Jolla, CA, 92093

Email: jrwilliams@ucsd.edu 


\begin{abstract}
Items that are held in visual working memory can guide attention towards matching features in the environment. Predominant theories propose that to guide attention, a memory item must be internally prioritized and given a special template status, which builds on the assumption that there are qualitatively distinct states in working memory. Here, we propose that no distinct states in working memory are necessary to explain why some items guide attention and others do not. Instead, we propose variations in attentional guidance arise because individual memories naturally vary in their representational fidelity, and only highly accurate memories automatically guide attention. Across a series of experiments and a simulation we show that (1) items in working memory vary naturally in representational fidelity; (2) attention is guided by all well-represented items, though frequently only one item is represented well enough to guide; and (3) no special working memory state for prioritized items is necessary to explain guidance. These findings challenge current models of attentional guidance and working memory and instead support a simpler account for how working memory and attention interact: only the representational fidelity of memories, which varies naturally between items, determines whether and how strongly a memory representation guides attention.
\end{abstract}

Keywords: visual working memory, attentional guidance, variable precision, internal attention

Public Significance Statement: When you are holding an item in mind (say, your red mug), your visual attention is automatically guided toward red information in the environment. However, this does not always occur, and seems to happen less often when you are holding in mind multiple pieces of information (say, your red mug and your blue coaster). This study demonstrates that the fidelity of a working memory representation alone may determine how strongly that item will interact with attention. Since memories vary randomly in fidelity and tend to be lower fidelity when holding more items in mind, this can explain why attention is sometimes, but not always, guided by items we hold in mind. 
As we look around the world, we have the sense of a rich and complete perception. This is in spite of the fact that we are only able to process a small fraction of the available sensory information. In order to effectively and efficiently operate within this sensory maelstrom, some of that information must be prioritized: either because it is physically more salient or because it matches our current task goals and intentions. For example, when looking for a friend in a crowd, we may carefully hold their distinctive visual features in mind-like the red shirt they are wearing - as we try to find a match, and our attention will be guided towards matching features in the environment. But what if that memory representation is inaccurate or noisy, and your friend's shirt is in fact more orange than red? Would guidance occur in this situation? Surprisingly, while attentional guidance has been studied extensively, the relationship between the fidelity of a memory representation and how effectively that item can guide attention is not well understood. Instead, most work has focused on the number of items that can guide attention and whether such items must have a special status within working memory, like being in the 'focus of attention'. Here we propose that representational fidelity of memories aloneindependently of any special status for an item in memory-is sufficient to explain why some items do and do not guide attention.

\section{Guidance by 1 vs 2 items}

It has been repeatedly found that when only a single feature is maintained in working memory, attention is automatically guided towards matching features in the environment (Olivers, Meijer, \& Theeuwes, 2006; Soto, Heinke, Humphreys, \& Blanco, 2005; Soto, Hodsoll, Rotshtein, \& Humphreys, 2008; Soto \& Humphreys, 2007, 2008). However, it is less clear whether multiple items held in working memory effectively guide attention in a similar way. At minimum, a significant literature suggests that guidance by multiple memory representations is more fragile than guidance by a single item, as often no guidance effects have been observed at all when 
participants remembered more than a single item (Frătescu, Moorselaar, \& Mathot, 2019; van Moorselaar, Theeuwes, \& Olivers, 2014; see Ort \& Olivers, 2020 for a review). Conversely, several studies report guidance when multiple items are held in mind, with some suggesting that attention is guided equally well by each memory item (B. Zhang, et al. 2011; B. Zhang, et al. 2018; Chen \& Du, 2017; Hollingworth \& Beck, 2016; Fan et al., 2019; Soto, \& Humphreys, 2008).

To date, these differing results have mostly been discussed in terms of limits in visual working memory by focusing on the number of remembered items that can be prioritized by attention and thus given a special template status (Chen \& Du, 2017; Fan et al., 2019; Frătescu et al., 2019; Hollingworth \& Beck, 2016; Olivers et al., 2011; van Moorselaar et al., 2014; B. Zhang et al., 2018). Specifically, attentional template accounts presume that working memory is organized into qualitatively distinct states, and propose that attention must be internally directed to a memory representation prior to it being able to guide attention (Olivers et al., 2011; van Moorselaar et al., 2014; Chen \& Du, 2017; Fan et al., 2019; Frătescu et al., 2019; Hollingworth \& Beck, 2016; Olivers et al., 2011; van Moorselaar et al., 2014; B. Zhang et al., 2018). This emphasis on the template status as being the most important component of guidance leads to the commonly debated question of how many items can achieve this privileged status since, under this framework, any other (non-attended) items cannot guide attention (Hollingworth \& Hwang, 2013; Olivers et al., 2011; van Moorselaar et al., 2014). This literature's focus on 'how many items' is consistent with the historically strong emphasis on quantifying visual working memory capacity by the number of representations that can be maintained (e.g., Cowan, 2001; Luck \& Vogel, 1997).

\section{Variation in memory fidelity}


A critical factor about attentional guidance, however, is that, for a remembered item to guide attention it must also be the case that it is an accurate and precise representation of the encoded item. Since attention is unlikely to be guided towards an item if the corresponding memory representation is weak, imprecise, or even focused on the wrong object, it is critical that we consider the quality of the memory representations themselves. All models of visual working memory capacity now acknowledge that items vary in strength or precision, such that representational fidelity tends to be higher when only 1 item must be held in mind than for 2 items (e.g., Zhang \& Luck, 2008; Bays et al. 2009). Additionally, many modern models see independent accumulation of noise across different items, and the resulting variation in memory fidelity across these items, as the core of visual working memory limits (Schurgin et al. 2020; Bays, 2015; Fougnie et al. 2012). Thus, even for very small set sizes, well within typical claims of 3-4 items "capacity limits" (Cowan, 2001), variation in representational fidelity appears to be an inevitable fact of working memory storage that must be accounted for when considering how working memory is used to guide attention.

Variation in representational fidelity is also not solely about the overall decrease in fidelity as more items must be held in mind. Individual items also vary in representational fidelity within a single trial. For example, Fougnie et al. (2012) found that when participants remember 3 colors, they are far more accurate at reporting a color of their choosing compared to when they report the color of a randomly probed item (similar results are found by Adam et al., 2017). This finding could only occur if the fidelity of remembered items varied considerably within a trial. Why might items vary in representational fidelity within a trial? This variability has been proposed to arise from many sources, including differential encoding precision (van den Berg et al. 2012), how memory items relate to other items on the encoding display (Brady \& Alvarez, 2015), differential prioritization through memory-related resource allocation (Bays \& Husain, 2008; Bays \& Taylor, 2018; Klyszejko, Rahmati, \& Curtis, 2014), and differences in the 
representation of specific individual colors (e.g., Morey et al. 2009; Bae et al. 2013). Variation in fidelity has also been proposed to be a basic fact about the architecture of the working memory system, with noise corrupting items independently (Fougnie et al., 2012; Bays, 2015; Schurgin et al. 2020; Panichello, DePasquale, Pillow, \& Buschman, 2018; Wilken \& Ma, 2004). These convergent results strongly suggest that variation between multiple working memory items is an inevitable and natural byproduct of working memory maintenance and is thus an important factor to consider for memory driven attentional guidance.

\section{Can attentional guidance be explained by memory fidelity?}

In the current work, we propose that variations in attentional guidance may be accounted for primarily by this variation in representational fidelity. While some previous work has investigated how memory fidelity relates to guidance, such studies have often concluded that guidance occurs for template items (Dube \& Al-Aidroos, 2019) and cannot occur for non-template items (Hollingworth \& Hwang, 2013), irrespective of how well they might be represented in memory. However, in our view, these studies do not allow strong conclusions about the relationship between guidance and memory strength, largely because of the way memory strength was measured. ${ }^{1}$ In contrast, in the current work, we directly test the relationship between memory strength and attentional guidance in a series of experiments that assess attentional guidance effects while precisely measuring memory strength of the remembered items. We ask participants to memorize two colors and use a simple search task that allows the detection of potentially small guidance effects. The implementation of a simple search task is in contrast to previous studies that have often used more complex search displays that may have taxed the

\footnotetext{
${ }^{1}$ In the discussion, we focus on why guidance studies in general, and why these studies in particular, are likely incapable of making strong conclusions about the relationship between memory strength and guidance.
} 
already limited attentional and working memory systems, perhaps, inadvertently obscuring a multiple item effect (Houtkamp \& Roelfsema, 2006; Hollingworth \& Hwang, 2013; Olivers et al., 2006; van Moorselaar et al., 2014; Woodman \& Luck, 2007).

We hypothesize that representational fidelity-how well a memory item accurately represents the initially encoded item - is sufficient on its own to determine whether and how strongly that item will guide attention. That is, guidance does not depend on the number of items that can be prioritized within working memory, but is rather determined by the representational fidelity of an item and the variation in representational fidelity between items that occurs naturally both across set sizes and within a single trial. We propose that the reason that guidance from multiple items is often found to be more fragile than single-item guidance is because it is less likely that multiple items are maintained in working memory with the strength and precision necessary to guide attention effectively.

\section{The current work}

Overall, across 3 main experiments and 2 supplemental experiments (see Appendix), we find evidence to support an account where items vary naturally in their representational fidelity, and any and all memory items can guide attention insofar as they are well represented-even without a special template status. By probing participants' self-selected memories and comparing them to randomly selected memory representations, in Experiment 1, we show that memories vary in their representational fidelity naturally, and that typical multiple-item guidance effects are primarily driven by the most well-represented memory. In a series of simulations, we show that the observed variation between remembered items is expected and well characterized by a working memory model that directly predicts how much variation should be present (via signal detection theory; Schurgin et al. 2020). In Experiment 2, by experimentally manipulating the supposed 'template status' of an item (with a retro-cue; van Moorselaar et al., 
2014) and the representational fidelity of items (adding low or high perceptual-noise at encoding), we show that differences in representational fidelity can explain variation in attentional guidance, with internal attention simply being one of many ways to boost the fidelity of the attended memory. Finally, in Experiment 3 we show that attention is guided by well represented memories even when those memories do not achieve a special 'template status' within working memory, finding guidance even for items placed outside 'the focus of attention'.

Our results can thus unify the seemingly irreconcilable findings that one or many working memory items can guide attention: When working memory resources are stretched among multiple active representations, often only a single item is represented well enough to guide (e.g., Exp. 1); however, in other cases, the representational fidelity of multiple items may be high enough to produce guidance from both items (Simulation, Exp 3, and Discussion). After presenting data from each experiment, we integrate across all of the experiments, and find compelling evidence that continuous variation in representational fidelity is sufficient to determine whether - and how strongly - an item guides attention, with no need to postulate distinct states in working memory, and that this accounts for guidance strength. Thus, these data support a model of memory-driven attentional guidance where representational fidelity fully explains how memories interact with attention and influence behavior. Overall, we conclude that (1) attention is guided by memories that accurately represent the encoded item, (2) that internal noise, accumulated independently for each item, determines whether an item is represented well enough to guide attention and (3) that no strict limits exist on the number of items capable of guiding attention; rather, any and all well-represented items can guide attention and that the magnitude of this guidance is directly related to an item's representational fidelity at that moment in time. We believe that this account explains and unifies many of the mixed results in the memory-driven attentional guidance literature and provides a new framework of how working memory and attention interact. 


\section{Experiment 1: Dissociating guidance for well-represented and poorly-represented working memory items}

Previous work has shown that memory driven attentional guidance is less strong when two items are maintained in working memory compared to one (e.g., van Moorselaar, Theeuwes, \& Olivers, 2014) and that representational fidelity varies considerably between actively maintained items in working memory tasks (e.g., Fougnie et al., 2012). We replicate these findings using our own paradigm in the Appendix, finding in Experiment A1 that guidance is less strong when two items are maintained than one; and finding in Experiment $\mathrm{A} 2$ that representational fidelity differs across memory items, such that 1 item tends to be maintained more accurately than the other when 2 items must be held in mind in an attentional guidance task (see Appendix).

In Experiment 1, we investigated whether this variance between multiple items in representational fidelity can explain the differences in guidance strength on any particular trial. In short, we asked whether the best represented item is generally responsible for the guidance effect by having participants perform both a search task and memory task on each trial. Participants maintain two unique colors in visual working memory for a memory task that occurred at the end of each trial. Prior to reporting one of the remembered colors on a continuous color wheel, participants performed a visual search task in which color was irrelevant. At the end of each trial, either one of the remembered items was randomly probed (forced report) or participants would freely report one item of their choosing (free report). Evidence suggests that people are nearly optimal at choosing the most precise item for report (Fougnie et al. 2012) and we hypothesized that, even without any explicit experimental manipulation-participants were simply instructed to report any memory item that they wishedindividuals would have knowledge about the representational fidelity of each item and, if some 
asymmetry between the items exists, would select the more precise item to report. Thus, free report trials allow us to estimate the representational fidelity of selected items, compare it to randomly probed items, and examine whether items differ in their representational fidelity naturally.

The primary goal of Experiment 1 is to relate the representational fidelity of individual items to the guidance effects during search. Specifically, by focusing on free report trials, we can sort trials based on whether a chosen memory item happened to be present in the search display that occurs before the memory report (chosen-item: present) or not (chosen-item: absent). Our critical condition here is chosen-item: absent, where we expect to find a diminished guidance effect (that could even be zero). This is because we expect items with less than optimal representational fidelity to exert limited guidance over attention. Chosen-item: absent trials should on average represent the amount of guidance that occurs from poorly represented items. By contrast, chosen-item: present trials likely represent a mixture of items that were well represented from the initial encoding (those that should guide) and trials where items are less well represented during the search task, but that are nonetheless chosen during the memory report, perhaps because of the brief re-exposure to that color during visual search². Nonetheless, any difference in guidance between these two trial types suggests that representational fidelity differences between the items are related to differences in attentional guidance.

\footnotetext{
${ }^{2}$ Note that while the search display is presented briefly so as to discourage intentional re-encoding, we have also replicated the same pattern of data in another experiment in which we used 2 set sizes (1 vs. 2) along with the free-report method, but had participants perform either search or the memory task on separate trials to avoid the possibility of re-encoding items during the search (see Appendix and Figure 10).
} 


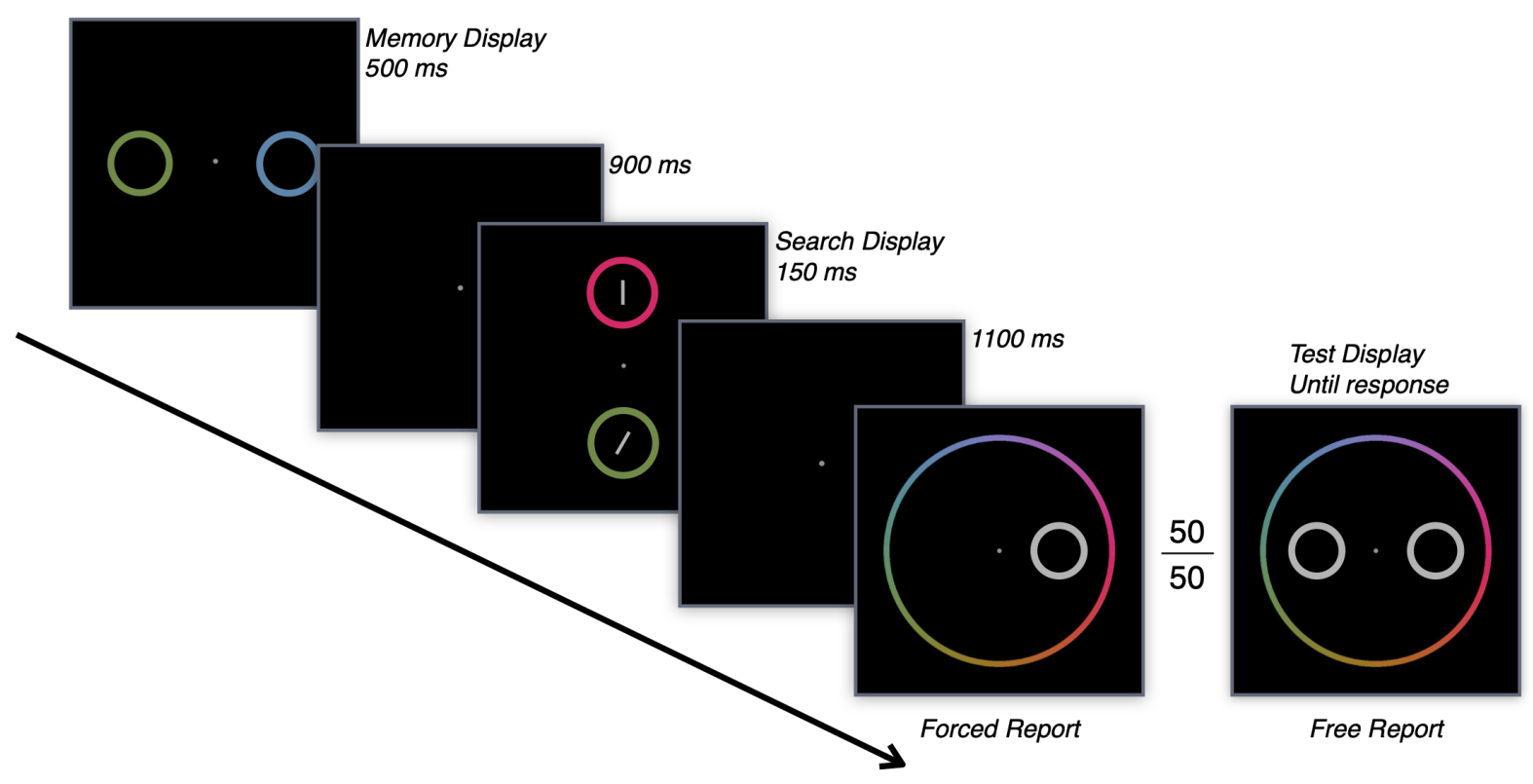

Figure 1. Experiment 1 Task design: Participants remember two colors on every trial, then perform a visual search task after a short delay, followed by a memory report at the end of each trial. On half of the trials participants are forced to report a particular item (left memory example), and on the remaining half of trials they can choose which color they want to report (free-report trials; right). This allows us to separately analyze the search benefit for free-report and random-probe memory conditions.

\section{Methods}

The design, sample size, exclusion criteria, and analysis plan for this experiment were pre-registered using AsPredicted (http://aspredicted.org/blind.php?x=7b5y74).

\section{Participants}

Consistent with our preregistration, the final sample included thirty participants (24 females) from UC San Diego, who took part in this study in exchange for course credit. Data from four additional participants were removed and replaced for failing to meet the preregistered exclusion criterion and, as in the Appendix experiments, data from another participant was removed and replaced for failing to follow forced-report instructions and thus failing to report the probed memory item on more than $40 \%$ of trials. 
Memory fidelity drives guidance

\section{Stimuli}

Stimuli were generated and presented using MATLAB and the Psychophysics Toolbox (Brainard,1997; Pelli, 1997). Memory items were colored rings that were $3^{\circ}$ visual angle in diameter, $0.3^{\circ}$ thick, and were centrally placed $4^{\circ}$ to the left or right of fixation. On every trial, the color of one memory item was randomly drawn from a uniformly spaced circle (radius $49^{\circ}$ ) extracted from the CIE $L^{*} a^{*} b$ space, centered at $(L=54, a=21.5, b=11.5)$ and when two memory items were present, the second color was selected to be $90^{\circ}$ away in color space from the first color (with $\pm 5^{\circ}$ of jitter). The search display consisted of a target line which was $0.3^{\circ}$ thick, $0.4^{\circ}$ long, tilted $0.06^{\circ}$ to the left or right of vertical, and placed $4^{\circ}$ above or below fixation and a single vertical distractor line that was placed at the opposite location (see Figure 1). The target and distractor lines were encircled in colored rings that matched one of the memory item properties. One of the colors matched a memory color and the other color was chosen to be $180^{\circ}$ away from it in color space (this was $90^{\circ}$ away from the other memory item). On the random probe memory display, one of the memory items initially appeared in gray (identical features to memory items) surrounded by a continuous color wheel which was $15^{\circ}$ in diameter, $0.3^{\circ}$ thick, and was centrally placed about fixation. On free report displays, two gray placeholders were presented and after one of these placeholders was selected, the continuous color wheel appeared.

\section{Procedure}

Each trial started with the presentation of two memory items $(500 \mathrm{~ms})$ that were to be remembered for a memory task at the end of the trial. Following a 900ms delay, a search display appeared for $150 \mathrm{~ms}$. The search display contained two lines, a distractor and a target line, above and below fixation. Participants needed to rapidly determine the orientation of the tilted, target line after the search display disappeared. Feedback to respond more quickly was provided when responses exceeded 1200ms. After participants provided a search response, 
and after a delay of $1,100 \mathrm{~ms}$ participants were probed on one of the memory items. On forced report trials a randomly selected memory item was presented in gray and would change color as participants moved the mouse around the continuous color wheel. On free report trials, participants were presented with two gray rings to the left and right of fixation. Here, they clicked on which item they would like to report prior to using the continuous color wheel (see Figure 1).

The memory task was evenly divided between the free report and forced report conditions and on half of all forced report trials the probed item was present in the visual search display and was absent on the remaining half. At test, participants were asked to use the mouse to find the color closest to the remembered color on the color wheel. The location of the testitem indicated which memory item should be reported (e.g., a test-item on the left probed the color of the memory item that was on the left at encoding), and which item was tested was counterbalanced across the experiment. Once the mouse was moved from the central fixation point the gray test-item changed color to match the color at the position of the mouse cursor. Once participants identified the color that matched the remembered color as precisely as possible on the color wheel, they locked their response by clicking the mouse button. Response error, defined as the difference in degrees between the provided response and the correct answer, was shown after every memory trial and participants were instructed to keep this error below $10^{\circ}$. Participants were instructed to prioritize speed without compromising accuracy for the search task and, for the memory task, were instructed to prioritize precision without compromising temporal efficiency.

\section{Analysis}

In the search task, we calculated each participant's median response time (RT) where the target was in the memory-matched color and in the distractor color separately. The difference between these two values was then taken as the amount of guidance for each 
condition. RTs that were faster than $200 \mathrm{~ms}$ or slower than $1500 \mathrm{~ms}$ were removed prior to any further analysis. All subsequent analyses use these criteria, unless otherwise noted.

Memory performance was evaluated in two ways. First, we used a simple descriptive statistic, angular deviation (a circular analog of standard deviation) to provide a nonparametric estimate of an item's representational fidelity. Second, we implemented the Target Confusability Competition (TCC) model to better quantify memory strength (Schurgin, Wixted \& Brady, 2020). The TCC model is based on recent evidence showing that continuous report memory distributions can be quantified by a single parameter - memory strength (i.e., d) - once the nonlinear nature of perceptual similarity is accounted for (Schurgin, et al., 2020). Specifically, for any given color wheel, there is a completely fixed perceptual similarity function that quantifies how confusable colors are, but this function is not linearly related to distance along the color wheel-rather, it is roughly exponential (as in Fechner's law).

Understanding this confusability function allows a simple signal detection model to explain working memory performance across a huge variety of conditions, with only a single parameter $\left(d^{\prime}\right)$. In particular, on any given trial, the to-be-remembered color is boosted by a strong familiarity signal (strength: $d$ ), and completely dissimilar colors do not have their familiarity signal boosted at all. Intermediate colors have their familiarity signals boosted proportional to how similar they are to the target. So, a color 1 degree away from the to-beremembered color gets a large boost in familiarity, and a color 10 degrees away from the to-beremembered color gets a moderate boost in familiarity. Noise is then added to these familiarity signals, and when participants are asked what color they saw, they report the color that has the highest familiarity.

Formally, this means the continuous report task is conceptualized as a 360-alternative forced choice task: Let $f(x)$ be how similar a given color is to the to-be-remembered color. Let 
$\left(\mathrm{X}_{-179}, \ldots, \mathrm{X}_{180}\right)$ be a vector of normal random values with means $d_{x}=d^{\prime} f(x)$ and unit variance. Then the reported color value, $r$, on this trial is simply:

$$
r \sim \operatorname{argmax}\left(\mathrm{X}_{-179}, \ldots, \mathrm{X}_{180}\right)
$$

In the current data, which uses the same color wheel used by Schurgin et al. (2020), we rely on their similarity data and their technique for fitting the model, including the necessary correlation between colors based on perceptual matching data to adjust d' (Schurgin et al. 2020).

Since the current study introduced a larger possibility of location confusions than the data fit by Schurgin et al. (2020), we introduce a "swap" parameter into the model (as in Bays, Catalao, \& Husain, 2009). In particular, rather than assuming participants always report based on the similarity, $f(x)$, to the target color, we assume that on some trials, participants instead respond based on similarity to the non-target (i.e., the item at the non-tested location). Let $f(x)$ be the similarity to the target color and $g(x)$ be the similarity to the non-target. Let $\left(\mathrm{X}_{-179}, \ldots\right.$, $\left.\mathrm{X}_{180}\right)$ be a normal random vector with means $d_{x}=d^{\prime} f(X)$ and unit variance, and $\left(\mathrm{Y}_{-179}, \ldots, \mathrm{Y}_{180}\right)$ be a normal random vector with means $d_{x}=d^{\prime} g(x)$, and unit variance. Let $\beta$ be the 'swap rate'. Responses are generated as follows:

$$
\begin{aligned}
& \mathrm{w} \sim \operatorname{Bernoulli}(\beta) \\
& r \sim w \operatorname{argmax}\left(\mathrm{Y}_{-179}, \ldots, \mathrm{Y}_{180}\right)+(1-w) \operatorname{argmax}\left(\mathrm{X}_{-179}, \ldots, \mathrm{X}_{180}\right)
\end{aligned}
$$

In other words, for each trial, participants report the maximum familiarity signal from either the target or non-target with some probability $\beta$ of reporting from the non-target distribution. We again make use of the Schurgin et al. (2020) similarity data and perceptual matching data.

Whereas for conditions with no swaps (e.g., single-item condition), d' alone provides a measure of how strong the memory was, in the presence of swaps it is unclear what to consider as the strength of the underlying memory. The d' parameter in the swap model is best thought of as "how strong memory would be if people always reported based on the correct target" (e.g., ignoring any contribution of swaps). This value thus only represents the actual memory strength 
across all trials if "swaps" occur totally based on response error, and even when participants misreport items, they always have a very strong representation of the correct target as well. By contrast, if participants tend to report an incorrect item as a form of "strategic guessing", e.g., selectively do so when they have very little information about the correct item (Pratte, 2019) then the best way to understand memory strength across all trials is to reduce the estimated $d^{\prime}$ by the proportion of swaps (e.g., assume on "swap" trials, people had $d$ ' $=\sim 0$ for the correct item). Telling apart these accounts-or where on this continuum people tend to be-is difficult and not our main purpose, and $d^{\prime}$ 'is an approximately interval measure, unlike percent correct or 'guess rate' (Macmillan \& Creelman, 2005). Thus, for our results, we report three measures of memory strength: angular deviation (just a descriptive statistic of how tightly clustered participants error are); $d^{\prime}$ (memory strength on trials where the correct item was reported); and adjusted $d^{\prime}$ (memory strength after adjusting downward to account for the possibility of no memories when participants made location swaps, e.g., $\left.d^{\prime}(1-\beta)\right)$. In general, all of our conclusions hold similarly for angular deviation and both $d^{\prime}$ 'measures, and thus hold regardless of what assumption is made about memory for the target on swap trials. 
A

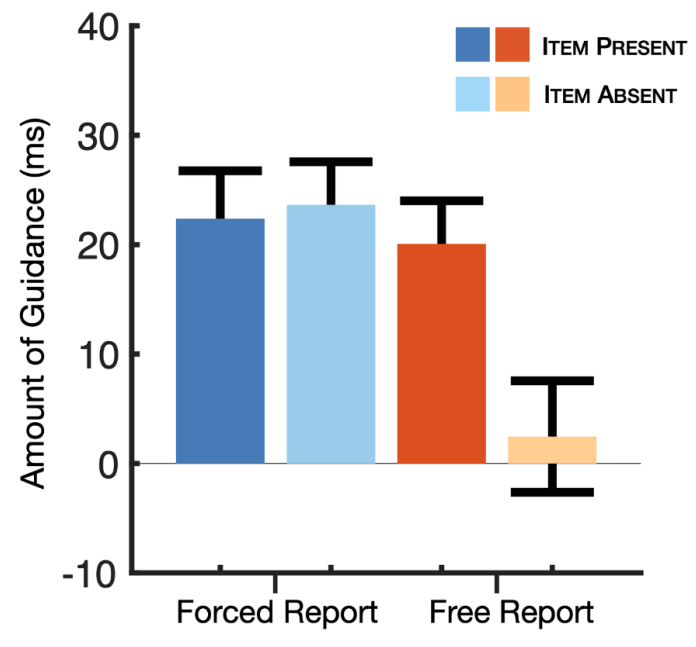

B
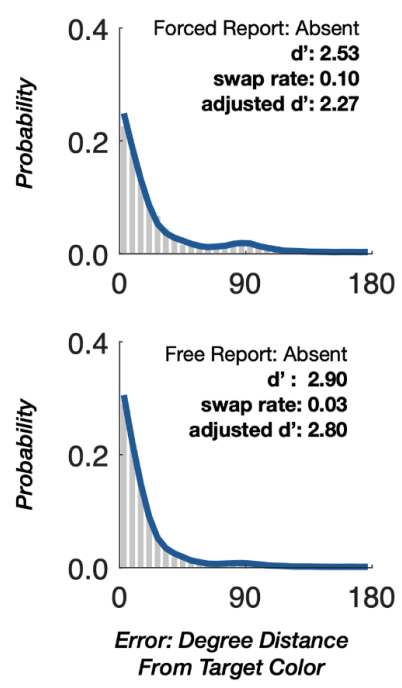
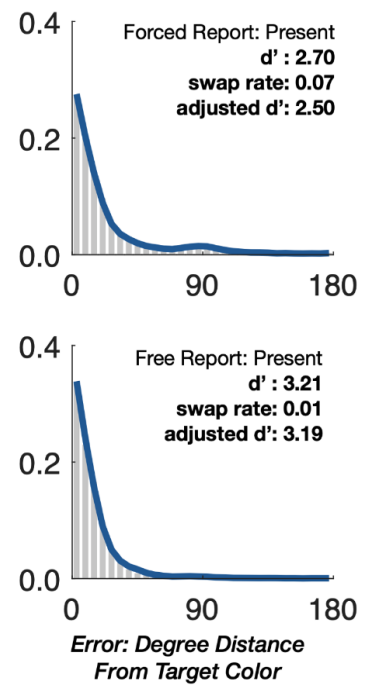

Figure 2. Experiment 1 results: A) Amount of guidance (RT for distractor minus target match trials) for forced report (blue bars) and free report (orange bars) trials separately for when the reported memory item was present or absent in the search display (dark vs light colors). For forced report trials, there is a clear guidance effect when the subsequently probed memory item was or was not present in the search display. For free report trials, by contrast, where participants are able to selectively report their strongest memory, there is a larger guidance effect when the subsequently chosen free report item was present in the search display than when the chosen item was absent. Thus, a memory item that is not subsequently chosen for report in free report exerts little to no influence over attention; resulting in a much smaller difference in RT for target and distractor match trials than a chosen item. B) Memory performance for forced report (top row) and free report (bottom row) separated by whether the reported item appeared in the search display or not. Memory strength was better on free report compared to forced report trials, suggesting participants report their strongest memories. In both cases, memory was slightly stronger when the relevant memory item was seen again on the search portion before the memory probe. Overall, then, memory was best when the freely chosen item was present on the previously encountered visual search display.

\section{Results \& Discussion}

Our main analysis focused on the magnitude of attentional guidance, operationalized as the difference in response time (RT) between match- and no-match trials, separately for each condition. We first submitted the average guidance effect, separated by memory condition (forced or free report) and search type (memory-item: present or absent), to a repeated measures ANOVA. This analysis revealed a significant main effect of memory condition $(F(1,29)$ 
$=4.19 p<0.05)$, no main effect of search type $(F(1,29)=2.09, p=0.16)$, and a significant interaction $(F(1,29)=7.73, p<0.01)$. Next, we examined the amount of guidance on forced report trials alone and found that participants were faster on target match compared to distractor match trials regardless of whether the search display contained an item that was subsequently tested $(t(29)=3.96, p<0.001)$ or not $(t(29)=4.46, p<0.001)$ and that the amount of guidance was consistent across these conditions as expected $(t(29)=0.21, p>0.05)$. To determine whether we have evidence to accept the null effect, we used Bayes Factors with a standard scale of the effect size (0.707) and the Jeffrey-Zellner-Siow Prior (JZS; Rouder et al., 2009). Here, we found that there is strong evidence to support no difference between these conditions $\left(B F_{01}=5.04\right)$.

Next, we examined the free-report condition alone. For chosen-item: present trials, we found that participants were significantly faster on trials where the chosen working memory item happened to match the color that surrounded the search target compared to distractor-match trials $(t(29)=3.6, p<0.001$; see Figure 2$)$. On the critical condition, chosen-item:absent, we tested whether unselected memory items would guide attention. Here, we found no significant difference between target- and distractor-match trials $(t(29)=0.37, p=0.72)$ and found evidence to support the null finding $\left(B F_{01}=4.84\right)$. Thus, the item that was not chosen in the memory task had little observable influence on visual search performance.

We next focused on memory performance to understand the relationship between guidance and memory strength. Memory performance was overall quite good and was better on free-report trials $\left(\operatorname{TCC} d^{\prime}=3.05\right.$, swap rate $=0.02$, adjusted $\left.d^{\prime}=3.00, s d=28.36^{\circ}\right)$ compared to random-probe trials $\left(\mathrm{TCC} d^{\prime}=2.62\right.$, swap rate $=0.08$, adjusted $d^{\prime}=2.38, s d=38.7^{\circ} ; t(29)=$ 7.37, $p<0.001)$, as expected. Memory performance was superior when the freely reported item was present in the previously seen visual search task $\left(\operatorname{TCC} d^{\prime}=3.21\right.$, swap rate $=0.01$, adjusted $\left.d^{\prime}=3.19, s d=26.25^{\circ}\right)$ compared to when it was not $\left(\right.$ TCC $d^{\prime}=2.90$, swap rate $=0.03$, 
adjusted $d^{\prime}=2.80, s d=30.47^{\circ} ; t(29)=3.36 p<0.01$, suggesting a benefit arose from reexposure. To check whether participants were biased to choose the memory item that was briefly presented during visual search more often than the absent item, we compared the proportion of chosen-items: present to chosen-item: absent trials. We found that participants reported the item that was absent from the search display about as often ( $47 \%$ of trials) as items that were present; which suggests that participants were not more likely to report an item that was present in the search display. Furthermore, when participants reported an absent item, they tended to have a very strong representation of it $\left(d^{\prime}=2.90\right)$ and rarely reported the wrong item (swap rate $=0.03$ ). Thus, participants were not biased to select an item that they had previously seen in the search display despite being briefly re-exposed (for $150 \mathrm{~ms}$ ) to that color.

Overall, in Experiment 1, we find that one item tends to be better represented than another, as free-report memory probes result in higher fidelity memories, and that less wellrepresented items are unlikely to guide attention. In particular, we find little to no evidence of attentional guidance by a memory representation that is not chosen by participants as their strongest memory. Additionally, when two items are maintained in working memory, we find that guidance can largely be explained by a single working memory item (similar to van Moorselaar et al., 2014; Beck \& Vickery, 2019). These results demonstrate that multiple items are represented with varying levels of representational fidelity and appear to exert correspondingly differential influences over attentional guidance.

\section{Simulation: Representational fidelity naturally varies between items even when all items are encoded equally well}

Experiment 1 revealed that working memory items naturally vary in how accurately participants can report them: Allowing participants to freely choose a memory item to report results in improved memory performance for the chosen item relative to a randomly probed 
item. Furthermore, the chosen item - the one with better memory performance-primarily guides attention, while the other, less precise item (that is not chosen) has little influence on visual search. While this shows a link between guidance and memory fidelity, it does not provide any evidence against a special template or focus of attention account. In particular, while the majority of models suppose stochasticity is sufficient to explain why memory fidelity varies (e.g., Schneegans \& Bays, 2020; Schurgin et al. 2020; Fougnie et al. 2012), another possible explanation for this variation in memory fidelity is that it too is caused by directing attention to one of the items-either externally, during encoding or internally, during the delay period, thereby giving some items a special status within the structure of working memory (e.g., Oberauer \& Lin, 2017). Such a model is based on the idea that working memory is divided into qualitatively distinct states, and that item(s) can achieve a special status, which results in a strong memory for that item and strong guidance during visual search (Olivers et al., 2011). Such an account is possible and consistent with the data so far but this model makes strong assumptions, namely that working memory consists of fundamentally different memory states.

In many ways, a more simple possibility is that items intrinsically vary in how accurately they reflect the encoded item, and that items that are poorly represented simply cannot effectively guide attention. Such between-item variation appears to arise naturally due to noise in memory that is independent for each item (e.g., Fougnie et al., 2012; Schurgin et al. 2020; Panichello, DePasquale, Pillow, \& Buschman, 2018). For example, Fougnie et al. (2012) found that while items vary in precision, this variation is not at all correlated across items - contrary to what you'd expect if there is some overarching attentional resource that is being unevenly distributed across items (as suggested by attentional template accounts).

In a series of simulations we answer two related questions: Is the amount of variation we find between the two items in memory reports consistent with this natural variation account? Or does it require additional assumptions about special attentional states within working memory? 

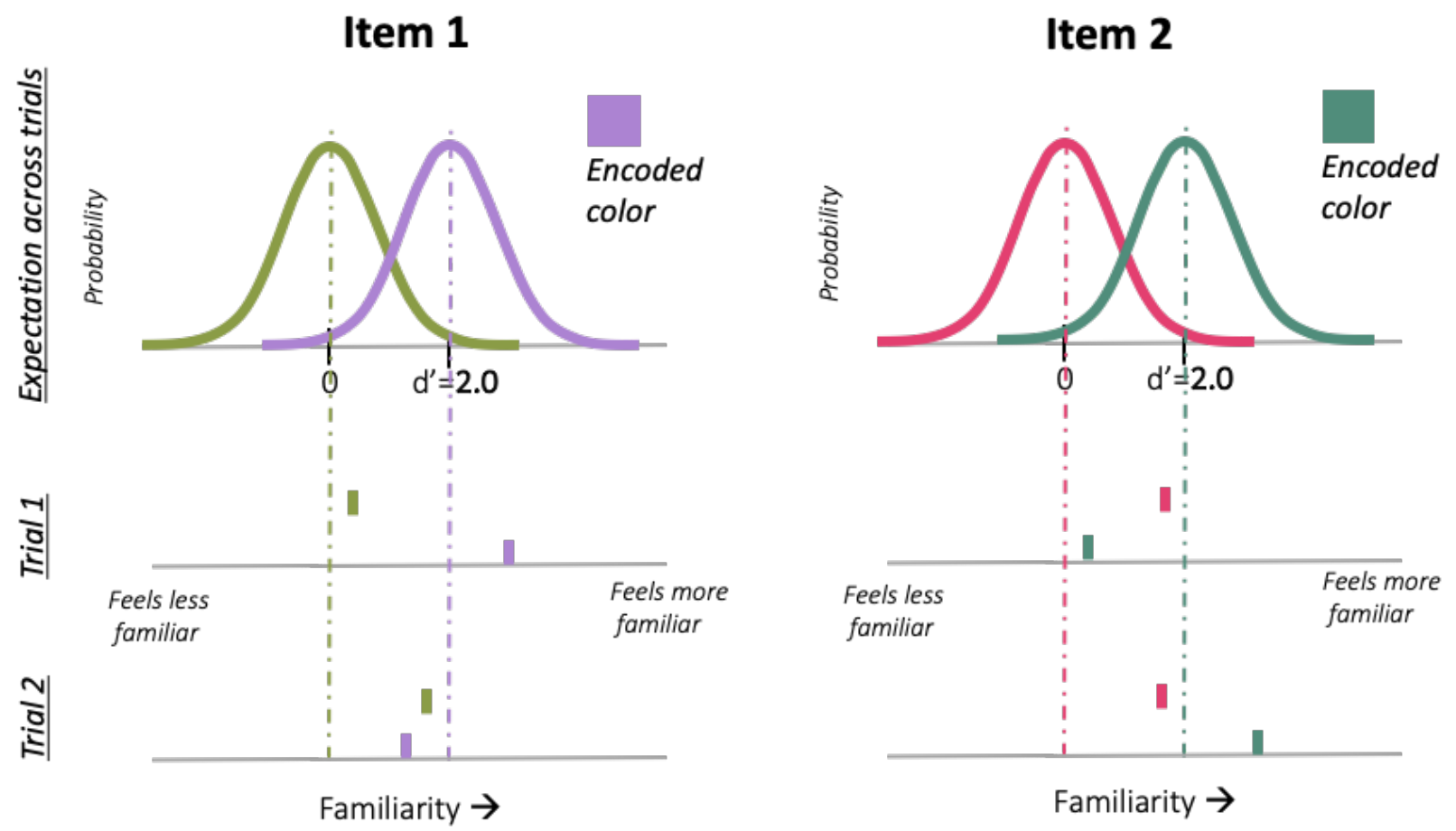

Figure 3. The signal detection account of memory. Imagine a scenario where people encode two items, one in one location and the other in a separate location and have independent memories for the two items. In this case, both items have the same underlying memory strength, in the sense that the signal to noise ratio for both is $d^{\prime}=2.0$ on average. That is, the encoded color is "boosted" in familiarity by two standard deviations $(d$ ' $=2.0)$ relative to how familiar an unseen, completely distinct color is (which is centered at familiarity $=0$ ). Thus, on average, participants find the color they actually saw at each location the most familiar. However, on any individual trial, the representation of one item may end up more or less accurately reflecting the original color due to independent noise. That is, signal detection conceives of each color for each item as varying in familiarity trial-to-trial (e.g., any given trial is a sample from the across trial distribution). Thus, the representational fidelity of an item varies from trial to trial from this noise process, even with the same underlying memory strength $(d)$ for both items. In signal detection, confidence arises from the same familiarity signal as the decision. Thus, whichever item ends up the strongest "winning" signal (e.g., the strength of the most familiar color) is the one we'd expect people to report in a free report task.

Importantly, variation between items doesn't need to be explicitly accounted for in the TCC model of Schurgin et al. (2020) that we use to fit memory distributions throughout the current paper, and despite this, this model makes precise predictions about how memory reports should vary between items, but it predicts this without explicitly modeling variation between items. Interitem variability in this model is simply as a natural consequence of a signal 
detection process (i.e., independent accumulation of noise for each item; see Figure 3). The expectation of noise or variability in the absence of an overarching attentional resource is common in models of memory and several models of working memory make precise and explicit quantitative predictions about how much variation we should expect between items at a given set size. For example, van den Berg et al. (2012) propose a particular mathematical relationship between variability and set size, with variability following a gamma distribution within set size and the mean of this distribution varying according to a power law across set sizes.

TCC, on the other hand, like all signal detection-based accounts of memory, proposes that the familiarity signals we use to decide which item we saw are inherently noisy. That is, even if you never saw a green item on a given trial, green might feel more familiar or less familiar (the top left of Figure 3 shows the probability distribution across trials of how likely green is to feel each level of familiarity). While actually seen items are on average more familiar than items you haven't seen (as reflected in a higher familiarity, on average shifted by $d^{\prime}=2.0$, of the purple item in Figure 3), they also vary in familiarity, such that they might feel more familiar (purple item on trial 1) or less familiar (purple item on trial 2 ) across trials.

Similarly, within a given trial, the TCC model proposes that item representations accumulate independent noise (Schurgin et al. 2020; see also, Fougnie et al. 2012). So, if you encode both a purple item and a green item, then on a particular trial, the purple item may happen to feel familiar, and the green item might feel less familiar. Importantly, simply knowing the underlying memory strength (d) of the items on average allows us to predict exactly how much they should vary trial to trial - because $d$ ' is a signal-to-noise ratio, we can use it to infer exactly how much variation in ultimate memory performance there should be between items that accumulate independent noise. The TCC model makes a slightly more complex prediction than the simple signal detection theory account, since this model predicts not just how likely people 
are to endorse having previously seen purple, but also exactly how likely they are to endorse any other color as the previously seen color. However, this same signal detection-based logic applies equally to this model, with the added idea that when you encode purple, not only does purple feel more familiar, but all similar colors also get enhanced familiarity.

In sum, this model says that while the noise is independent across different items, within the representation of an item, the familiarity signal is not independent for each of the colors but depends on the underlying perceptual similarity structure: if purple is encoded, not only does purple get a boost in familiarity (d), but similar colors (e.g., pink) get a boost, more so than distinct colors (e.g., yellow; see Figure 4 and Methods from Exp. 1). When we add noise, this makes participants more or less likely to endorse particular colors as the most familiar, and this differentially impacts different items.

\section{Item 1}

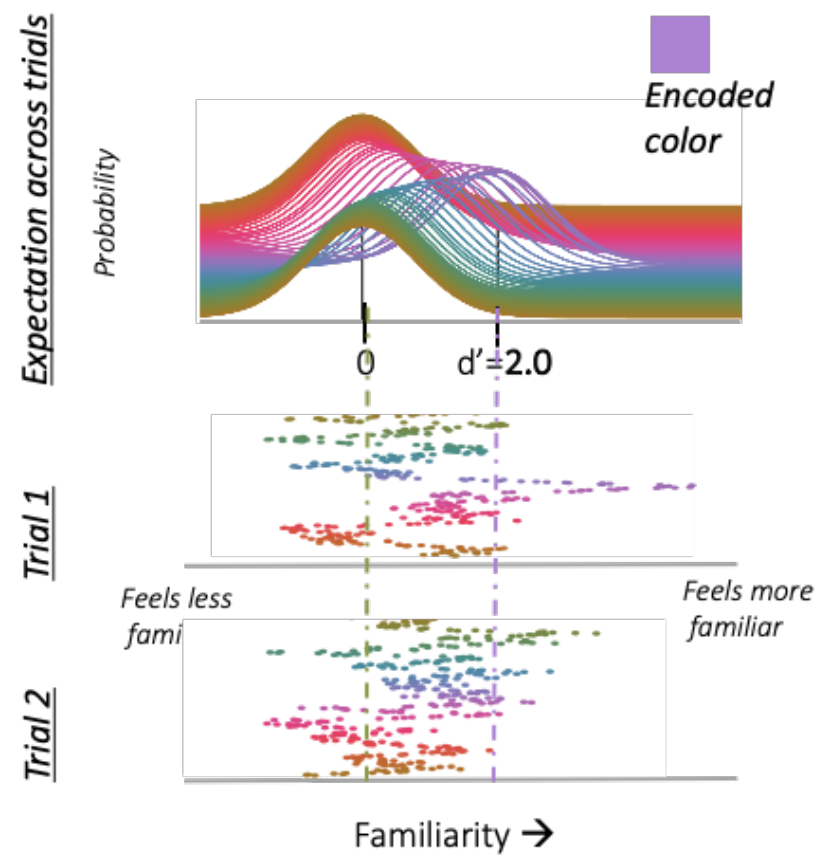

Item 2

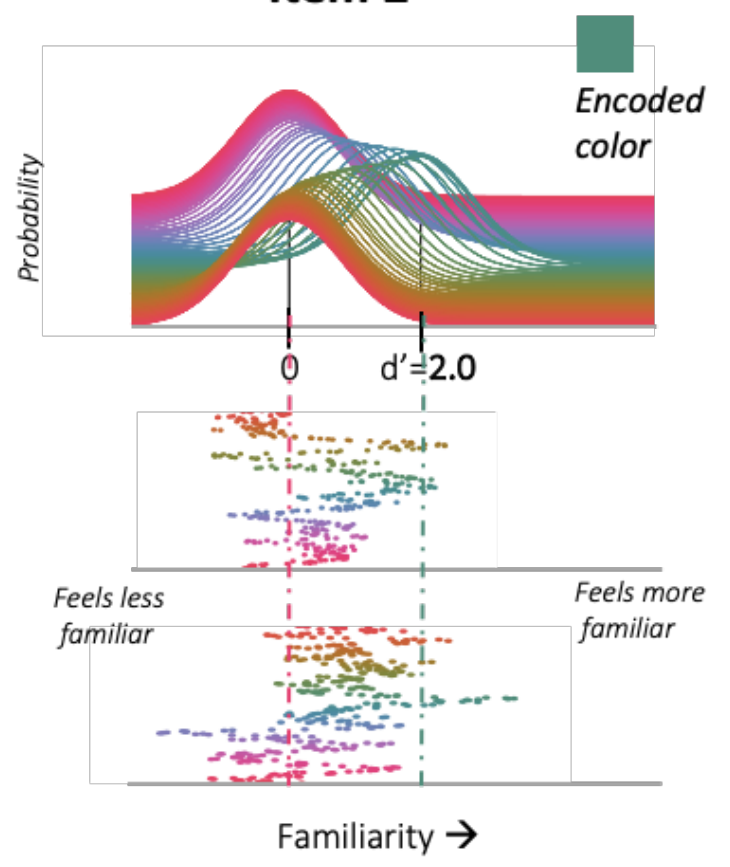

Figure 4. In the Target Confusability Competition model (TCC), the familiarity signal for particular colors depends on the fixed underlying similarity structure of the color representations. When one color is encoded, not only that particular color gets a boost in familiarity (of $d$ ), but 
colors that are perceptually similar to that color are also enhanced and thus more likely to feel familiar relative to distinct colors. Added noise on individual trials results in differential representational fidelity across items within a trial, even when each item has the same initial memory strength $\left(d^{\prime}\right)$. For example, on Trial 1, this individual has a lot of confidence that the purple item is some kind of purple, whereas the most activated color channel for the green item is yellow, so on this trial, the representational fidelity of Item 1 is greater than that of Item 2, even though both have the same underlying signal-to-noise ratio (d).

How does this a priori prediction of how much items should vary in memory performance relate to the actual variability observed in the free report condition of Experiment 1 ? To test this, we take the $d$ ' estimated from the overall average performance across items on forced report trials and use this to simulate (1) how much variability we expect between items in terms of performance, and (2) how this relates to actual free report performance. In particular, we assume that during initial encoding, all memory items are encoded equally well (with the same d), resulting in the same familiarity boost of the to-be-remembered color and similar colors (see Figure 4). Then, during the delay period, noise is added to all color representations, separately for each item, which changes the familiarity signals for each of these color representations.

In a standard forced probe situation, participants report the color they find to be most familiar for the probed item. In the free report condition, they consider the most familiar color from both items, and choose to report the color for the item that has a higher familiarity. We can then ask whether the amount of variation we observe in our data-e.g., the improvement of memory reports in free report trials relative to forced choice trials -is consistent with the variation predicted by this framework, or whether it exceeds it and thus calls for another explanation (like a special attentional focus that biases item representations systematically). 
A

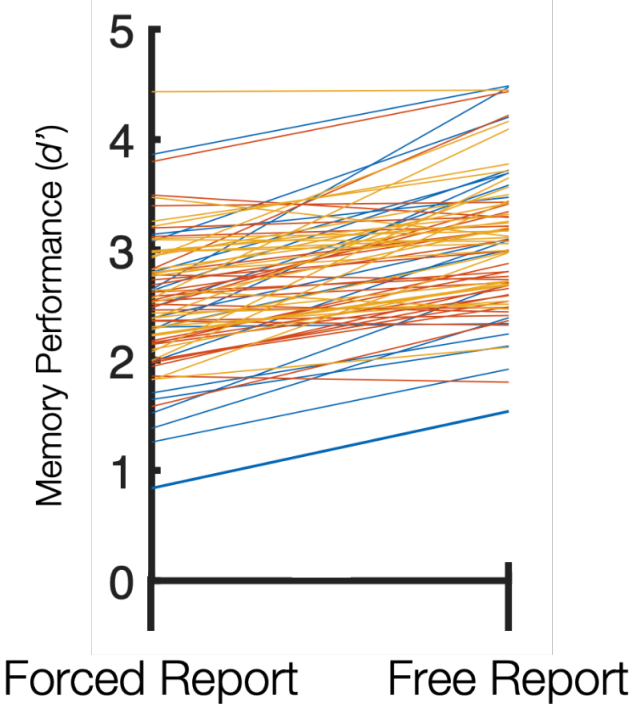

B

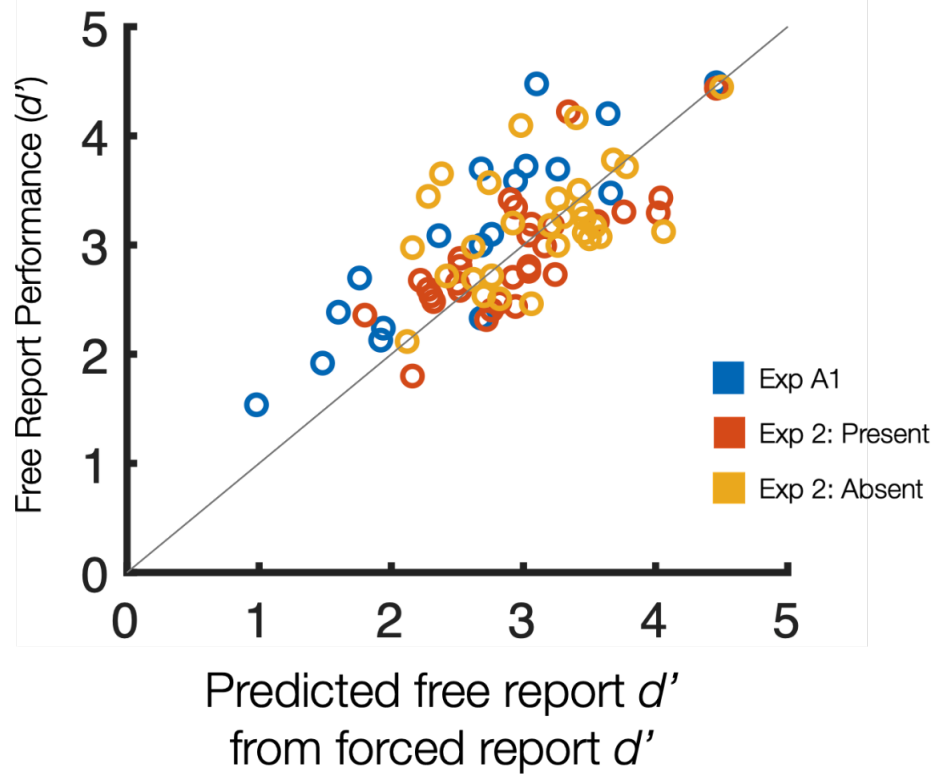

Figure 5. (A) Individual lines are subjects: this shows that free report reliably exceeds forced report across all experiments. (B) Signal-detection based prediction about free report with the strong assumption that all of the variability comes simply from the independent noise added to each item: e.g., with the assumption that d' for both items is exactly what is estimated from forced report (and thus the same initially), and the only difference in free report is that people report their most confident memory (e.g., the signal that has the strongest familiarity on that trial). This provides an excellent explanation of free report performance, with no actual difference between the items other than the noise process predicted by signal detection.

Figure 5A shows that across both Experiment 1 and Experiment $A 2$ from the Appendix, free report results in reliably higher memory performance than forced report. As shown in Figure $5 \mathrm{~B}$, we find that this difference between free report and forced report matches the prediction that both items were encoded with the same signal and had a similar amount of noise added to them (as reflected by the data being centered on the diagonal; $r=0.73, p<0.0001$ ). Thus, the variability between items that necessarily arises from the signal detection process is sufficient to explain the variability in memory performance that we observe. This demonstrates that assuming intrinsic variation in the representational fidelity of memories-due to independent item noise-is sufficient to explain the difference in memory performance we observed in our previous experiment. This assumption is also consistent with data indicating items vary in 
precision independently of each other (Fougnie et al. 2012). Overall, our simulations argue that attentional guidance is driven by an item that happens to have a stronger representational fidelity - and most accurately represents the initially encoded color - on that trial simply as a result of natural variation, and without needing to confer any special status on an item.

\section{Experiment 2: Effects of attentional cues and memory fidelity on search}

Our data, together with the simulations, are consistent with a representational fidelity account. In particular, memory representations vary naturally due to noise, producing asymmetries in representational fidelity between items, and that guidance effects are present when the item that happens to have a strong memory representation on a given trial is present in the search display. This explanation differs from attentional template frameworks which embrace distinct states among working memory items and further propose that attention is exclusively guided by any item that achieves a special and prioritized template status. Critically, such theories maintain that the template status is the single most important factor to predicting guidance and report that the precision of template item(s) has little (Frătescu et al., 2019; van Moorselaar et al., 2014) to no impact on attentional guidance (Dube \& Al-Aidroos, 2019; Rajsic, Ouslis, Wilson, \& Pratt, 2017; Hollingworth \& Hwang, 2013; B. Zhang, et al., 2018; Fan et al., 2019; Hollingworth \& Beck, 2016; B. Zhang, et al., 2018; but see Hout \& Goldinger, 2014).

For example, Dube \& Al-Aidroos (2019) found that a 100\% valid attentional retro-cue resulted in attentional guidance, but that a $70 \%$ valid cue did not; despite producing indistinguishable memory performance between the two conditions. They concluded that memory strength alone is not sufficient to grant a memory item with the template status and thus guide attention. Similarly, Hollingworth \& Hwang (2013) report that an uncued item does not guide attention irrespective of being very well-represented and suggested that this is because the item lacked a template status (a finding which is contradicted by B. Zhang, et al., 
2018). Yet, here, we have shown that memory strength is highly predictive of search by demonstrating that well-represented memories guide attention and that less well-represented items do not (Exp. 1), and that the focus of attention is not necessary to explain the variation in memory fidelity (Simulation). Our results thus far are consistent with the proposition that representational fidelity primarily determines whether and the extent to which an item will guide attention: as a representation becomes less and less identical to the initially encoded item, it will exert guidance over attention that is equally less and less efficacious.

In Experiment 2, we asked whether memory performance ( $d$ ', an aggregate measure of representational fidelity), or a special focus of attention better predicts guidance effects. In most studies, including our Experiment 1, it has been difficult to estimate representational fidelity and attention separately since attended items are usually maintained more precisely than other working memory items (see Rajsic et al., 2017; Oberauer \& Lin, 2017). Thus, we next separated the influence of representational fidelity and attentional focus on guidance by independently varying attentional focus and representational fidelity across trials. Participants performed a similar task to before, except now, to manipulate representational fidelity, we added different amounts of perceptual noise during encoding. This has been shown to increase confusability between colors, thus decreasing the signal to noise ratio of the memory representations without manipulating attention (see W. Zhang \& Luck, 2008). We also varied attentional focus using a directional retro-cue which has been shown to change the attentional state and improve the representational fidelity of an item by protecting it from interference (e.g., Oberauer, 2002; Oberauer \& Lin, 2017) $)^{3}$.

\footnotetext{
${ }^{3}$ Note that while some purport that the retro-cue facilitates memory performance by giving it a special status (i.e., placing it within the focus of attention), it is equally likely that retro-cue effects arise from the flexible allocation of a continuous memory resource (see Bays \& Taylor, 2018)
} 
The goal of Experiment 2 is thus to change the representational fidelity of memory items without changing the focus of attention, and vice versa, and to test whether differences in fidelity can affect visual search performance, independently of an item's attentional status. If memory fidelity plays an important role, guidance should vary according to how well an item is represented. And, if attention is simply one way of many to modulate memory fidelity we'd expect guidance to be greater for attended items but still vary depending on the representational fidelity at the time of search. If, however, memory fidelity plays little to no role, as previously stated by attentional template accounts (Dube \& Al-Aidroos, 2019; Rajsic, Ouslis, Wilson, \& Pratt, 2017; Hollingworth \& Hwang, 2013; B. Zhang, et al., 2018; Fan et al., 2019; Hollingworth \& Beck, 2016), once an item has achieved template status by being focally attended, then we should find a similar sized guidance effect across all items with a template status, regardless of their memory fidelity. 


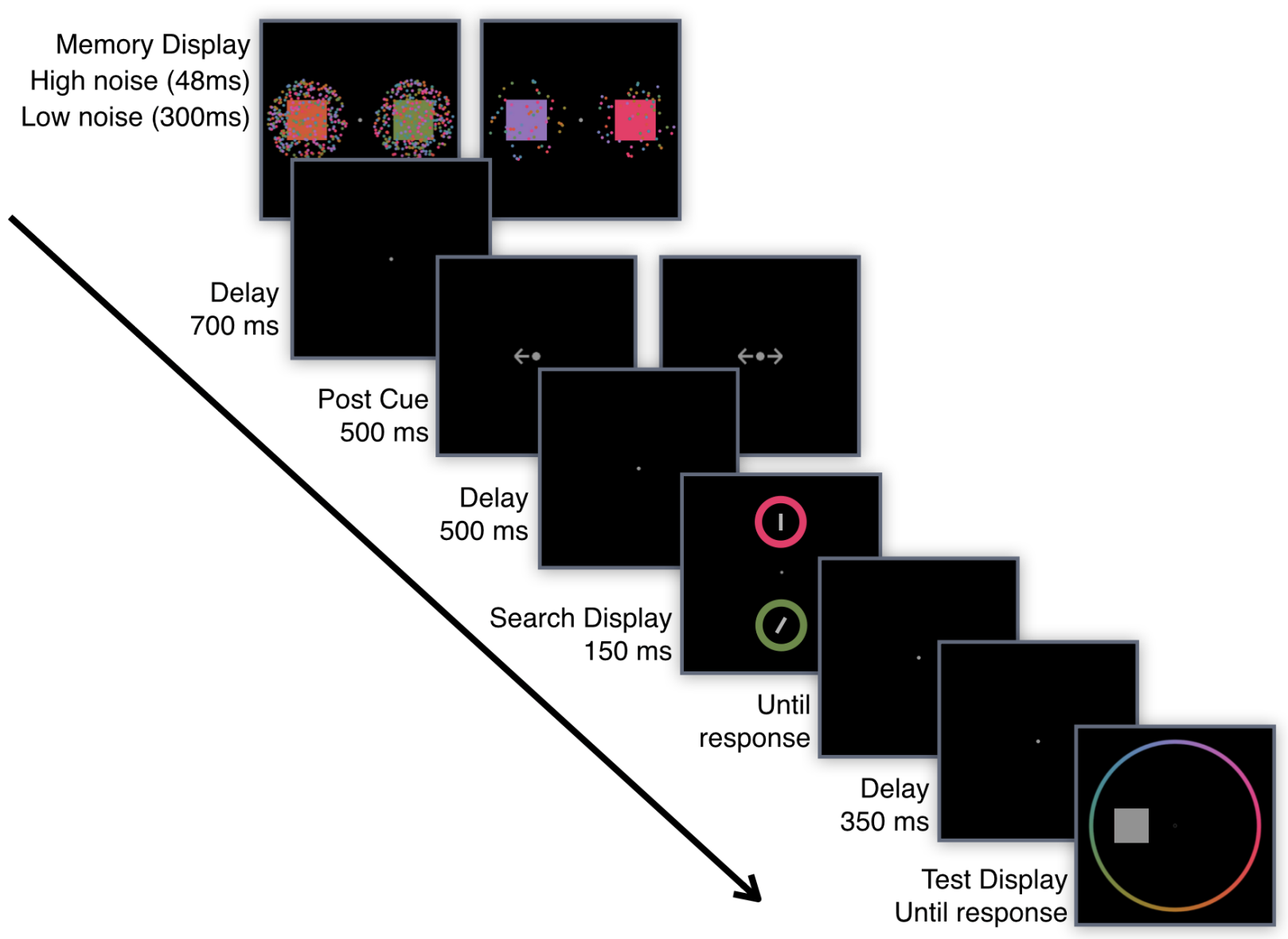

Figure 6. Experiment 2 task design: Participants remembered two items on every trial. Memory performance was manipulated in two ways: First, by presenting the items with many colored dots for a short time (high-noise trials; left) or a few colored dots for a long time (low-noise trials; right); more perceptual noise at encoding increases an item's confusability with other colors. Second, we presented a post-cue (i.e., retro-cue) during the delay period that was either neutral (distributed-attention condition; right) or directed to one of the items with $100 \%$ validity (directedattention condition; left). This attention manipulation determines which item is in the 'focus of attention'. These manipulations allowed us to modify representational fidelity and attentional focus relatively independently. Finally, each trial continued with a search task, followed by the memory report task.

\section{Methods}

The design, sample size, exclusion criteria, and analysis plan for this experiment were preregistered using AsPredicted (https://aspredicted.org/blind.php?x=xx5wr8). 
Memory fidelity drives guidance

\section{Participants}

The final sample included 50 participants from UC San Diego who took part in this study in exchange for course credit. Data from an additional eight participants were removed for failing to meet the predefined inclusion criteria and, as in the previous experiments, data from three others were removed for incorrectly reporting the probed memory item on at least $40 \%$ of trials (greater than 3 standard deviations from the group average).

\section{Stimuli}

Memory items were presented as squares with a side length of $3^{\circ}$ in visual angle. On high-perceptual-noise (high-noise) trials, 360 uniquely colored dots (each $0.23^{\circ}$ in diameter) were randomly positioned within an invisible circle $\left(6^{\circ}\right.$ in diameter) over each memory item. On low-perceptual-noise (low-noise) trials, 60 uniquely colored dots were randomly positioned over both memory items. To achieve an even distribution of colors within the perceptual noise, the colors of the dots were chosen to be $1^{\circ}$ and $6^{\circ}$ apart in color space for high- and low-noise trials respectively. Post-cue displays consisted of either a neutral-cue (two arrows, each facing away from fixation and towards the memory items) or a directional-cue (one arrow facing one of the memory items; each arrow was $0.64^{\circ}$ long and $0.1^{\circ}$ thick). The memory report display was identical to previous experiments except that the test-item(s) were squares with equal proportions as the memory items.

\section{Procedure}

High-noise displays were presented for $48 \mathrm{~ms}$ while low-noise displays were presented for $300 \mathrm{~ms}$. Following the presentation of the memory items, there was a $700 \mathrm{~ms}$ delay. After this delay, on 256 trials for both high- and low-noise trials, participants were shown a neutral postcue; on the other 256 trials, they were shown a directional post-cue which cued them to the item that was to be probed in the final memory task with $100 \%$ validity. After a further delay of 500ms, participants performed the visual search task. After the search task-briefly flashed for 
$150 \mathrm{~ms}$ and followed by an untimed response window-and a further delay of 350ms, the memory report display was presented. On neutral cue trials, there was a $50 \%$ chance of each item being probed and on a directional cue trial the cued item was always tested. On both kinds of trials, $50 \%$ of the time the subsequently-tested item was the memory-matched color from the visual search display and on $50 \%$ of these trials, the memory-match color was the visual search target, and on $50 \%$ of these trials the memory-match item was the distractor (see Figure 6).

\section{Model fitting}

Note that in theory, adding noise will change the similarity function used by the TCC model to estimate memory performance (d'), because we are changing the stimuli themselves, and thus changing the similarity structure of the stimulus space (Schurgin et al. 2020). In particular, if the noise makes colors more perceptually confusable, the central part of the error distribution might be wider with noise than no noise (as observed by W. Zhang \& Luck, 2008). However, insofar as we are adding small amounts of color noise relative to the size of the stimulus, we assume these effects are small and continue to use the same color similarity function in the current data. This results in a good fit to the data (see Figure 7), suggesting the difference in similarity function is likely small with this level of noise. It may be that adding uniform color noise can be conceived of as simply increasing the familiarity of all of the colors present in the noise, and so with uniformly distributed noise, this is approximately equivalent to decreasing d'. 
A
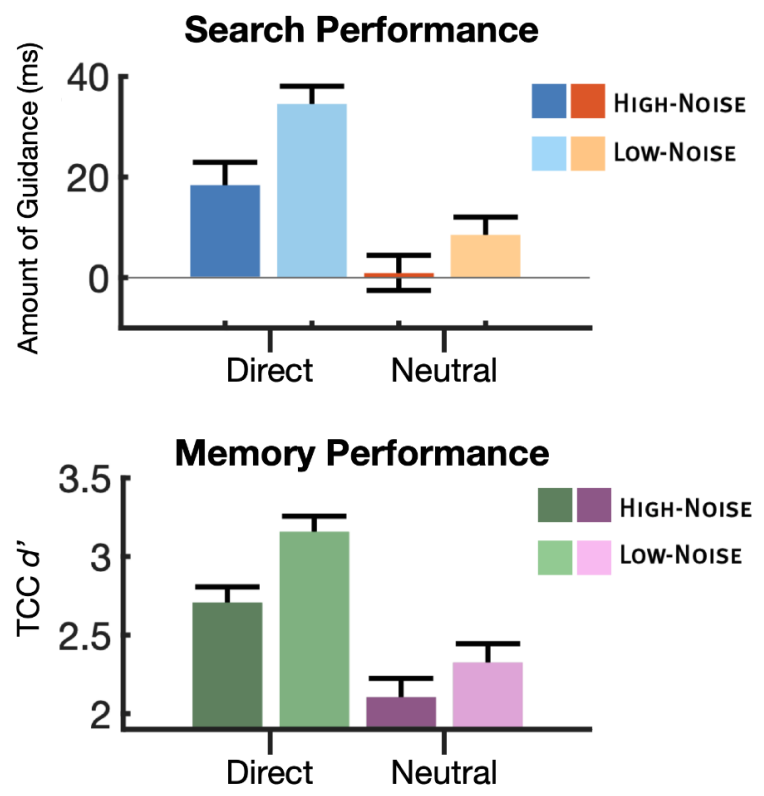

B
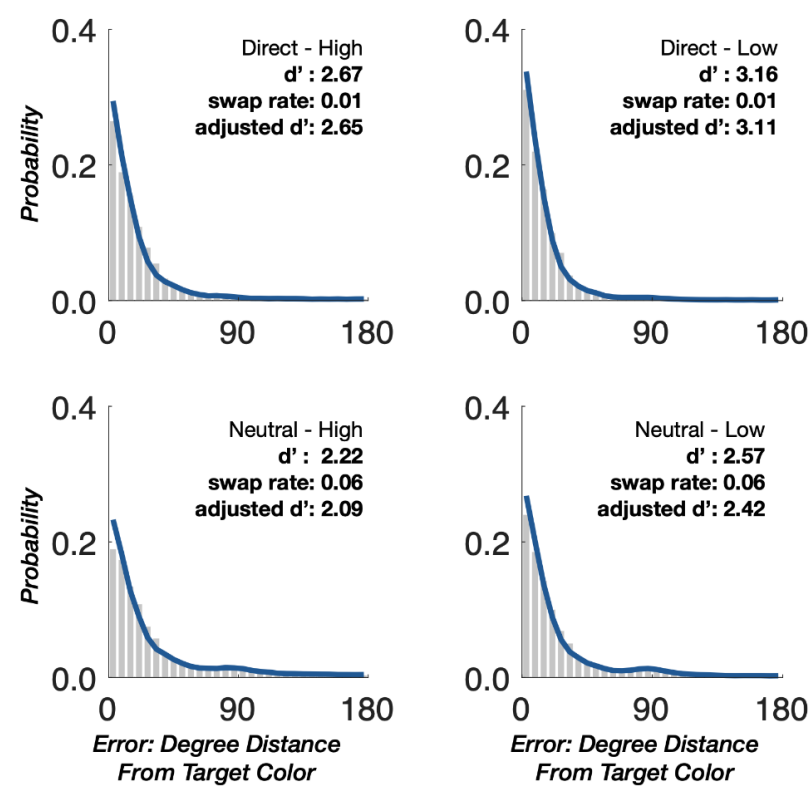

Figure 7. Experiment 2 results A) Top: Search effect (difference in RT between target and distractor match trials) for each of the four tested-item-present conditions (trials of which we have both memory and search performance). When an item was placed within the focus of attention by a directional retro-cue (blue bars) it biased attention in a graded fashion; more precise memory representations (low noise) resulted in a larger search effect compared to less precise representations (high noise). When no direct, attentional cue was present (neutral cue), the guidance effect was small overall. Bottom: Memory performance was highest for directionalcue trials with low-noise, followed by directional-cue trials with high-noise, neutral cue trials with low-noise, and finally neutral cues with high-noise. These data show that both - the attentional cue and the perceptual noise at encoding modulated memory performance, and that this was directly related to the amount of guidance observed; consistent with a representational fidelity account rather than a special focus of attention. B) Corresponding error histograms and TCC model fits for all conditions.

\section{Results \& Discussion}

As predicted, manipulating attentional focus and perceptual noise at encoding significantly influenced memory performance. Memory performance $(d)$ was submitted to a repeated measures ANOVA. The main effects of cue condition $(F(1,49)=182.81, p<0.001)$, noise condition $(F(1,49)=131.98, p<0.001)$, and search type (whether a tested memory item appeared in the search display; $F(1,49)=56.59, p<0.001)$ were significant. The interaction between the attention cue and noise conditions was also significant $(F(1,197)=17.16, p<$ 
0.001). Further analysis showed that average memory performance on trials with a directional cue $\left(\mathrm{TCC} d^{\prime}=2.91\right.$, swap rate $=0.01$, adjusted $d^{\prime}=2.89$, circular $\left.s d=25.97^{\circ}\right)$ was superior to those with a neutral cue $\left(\operatorname{TCC} d^{\prime}=2.39\right.$, swap rate $=0.058$, adjusted $d^{\prime}=2.26$, circular sd $=$ $34.05^{\circ} ; t(49)=11.46, p<0.001 ;$ Figure $\left.7 \mathrm{~B}\right)$, indicating that a retro-cue is effective in increasing average precision of remembered items. Additionally, when an item was cued, memory performance was modulated by perceptual noise at encoding such that memory performance was higher when a low-noise item was encoded (TCC $d^{\prime}=3.16$, swap rate $=0.013$, adjusted d' $=3.11$, circular $\left.\mathrm{sd}=23.8^{\circ}\right)$ compared to a high-noise item $\left(\operatorname{TCC} d^{\prime}=2.67\right.$, swap rate $=0.006$, adjusted $d^{\prime}=2.65$, circular $\left.s d=28.05^{\circ}\right)$ and the difference between them was significant $(t(49)$ $=9.89, p<0.001)$. Memory performance was also affected by perceptual noise on neutral cue trials such that low noise trials resulted in better performance $\left(\operatorname{TCC} d^{\prime}=2.57\right.$, swap rate $=0.059$, adjusted $d^{\prime}=2.42$, circular $s d=35.41^{\circ}$ ) compared to high noise trials $\left(\right.$ TCC $d^{\prime}=2.22$, swap rate $=0.055$, adjusted $d^{\prime}=2.10$, circular $\left.s d=38.7^{\circ} ; t(49)=8.24, p<0.001\right)$.

Next, we looked at search performance and found that attention was most strongly guided by items with the highest memory quality. We submitted the search effect from testeditem-present trials to a repeated-measures ANOVA with retro-cue (neutral, directional) and perceptual noise (high-noise, low-noise) conditions. This analysis showed significant main effects of post-cue $(F(1,49)=13.13, p<0.001)$ perceptual noise $(F(1,49)=4.8, p<0.05)$, and no interaction $(F(1,49)=0.61, p>0.05)$. We performed planned follow-up t-tests to better characterize the search effect for items within the focus of attention, that were both searched and probed at the end of the trial, and found that participants were faster on target-match trials compared to distractor-match trials regardless of whether high-noise items $(t(49)=2.46, p<$ $0.01, d=0.35)$ or low-noise items $(t(49)=5.50, p<0.001, d=0.78)$ were maintained. When attention was distributed between memory items we found a small search effect that failed to reach significance when a high noise item $\left(t(49)=1.51, p=0.14, d=0.02, B F_{01}=2.25\right)$ and a 
marginally non-significant effect when a low-noise item was maintained in working memory $\left(t(49)=1.89, p=0.06, d=0.26, B F_{01}=1.25\right)$.

Next, we looked at the difference in search effect across the directed attention conditions and found a reliable difference between high- and low-noise trials $(t(49)=2.17, p<0.05)$. Given that these two trial types resulted in differential memory performance, with low-noise trials having better memory performance $\left(\operatorname{TCC} d^{\prime}=3.16\right)$ relative to high-noise trials $\left(\right.$ TCC $\left.d^{\prime}=2.67\right)$, this indicates that the quality of the memory representation-above and beyond attention alone-is important in determining the amount of guidance. This finding demonstrates that a template item guides attention in accordance with its representational fidelity. This is in contrast to what would be expected if 'template status' per se was all that was critical to guidance.

While the results from this experiment contradict the strongest versions of the attentional template account wherein an item's status alone determines which item guides attention (Hollingworth \& Hwang, 2013; Fan et al., 2019; B. Zhang et al., 2011, 2018) it could be that the template status is a binary determinant of whether an item will guide at all, and memory fidelity subsequently determines the strength of guidance. To more explicitly determine whether the attentional template is a necessary function of attentional guidance, we next investigate whether an item without a template status guides attention if it is represented with high fidelity in memory.

\section{Experiment 3: Effects of representational fidelity on items outside the focus of attention}

In a final experiment we tested whether an item that is not directly attended can guide attention, as long as its representational fidelity is sufficiently high; a prediction that is directly contradictory to attentional template accounts. To more directly compare the effects of representational fidelity (by adding noise at encoding) and attentional status (through a retrocue) we manipulated both factors within the same trial. Attentional template accounts would 
predict no guidance for non-template items, whereas a representational fidelity account would predict that any well represented item exerts influence over attention.

Participants maintained one high-noise and one low-noise item in memory on the same trial and were subsequently retro-cued as to which item would most likely be tested on memory probe trials. Since it could be argued that memory performance is contaminated by the reappearance of one memory item in the search display, and this in turn may change participants' strategy during visual search and possibly interfere with the guidance effect, we now tested memory and search on separate trials (similar to our preliminary Experiments A1-2; see Appendix). This change eliminates any strategic attempt to improve memory by attending to the colors in the search display since participants will only perform one task per trial. We are particularly interested in whether a non-cued, and thus non-template item can guide attention when it is well represented, and whether, in general, memory fidelity tracks guidance, as we have seen in our previous experiments, and as predicted by our representational fidelity hypothesis. 


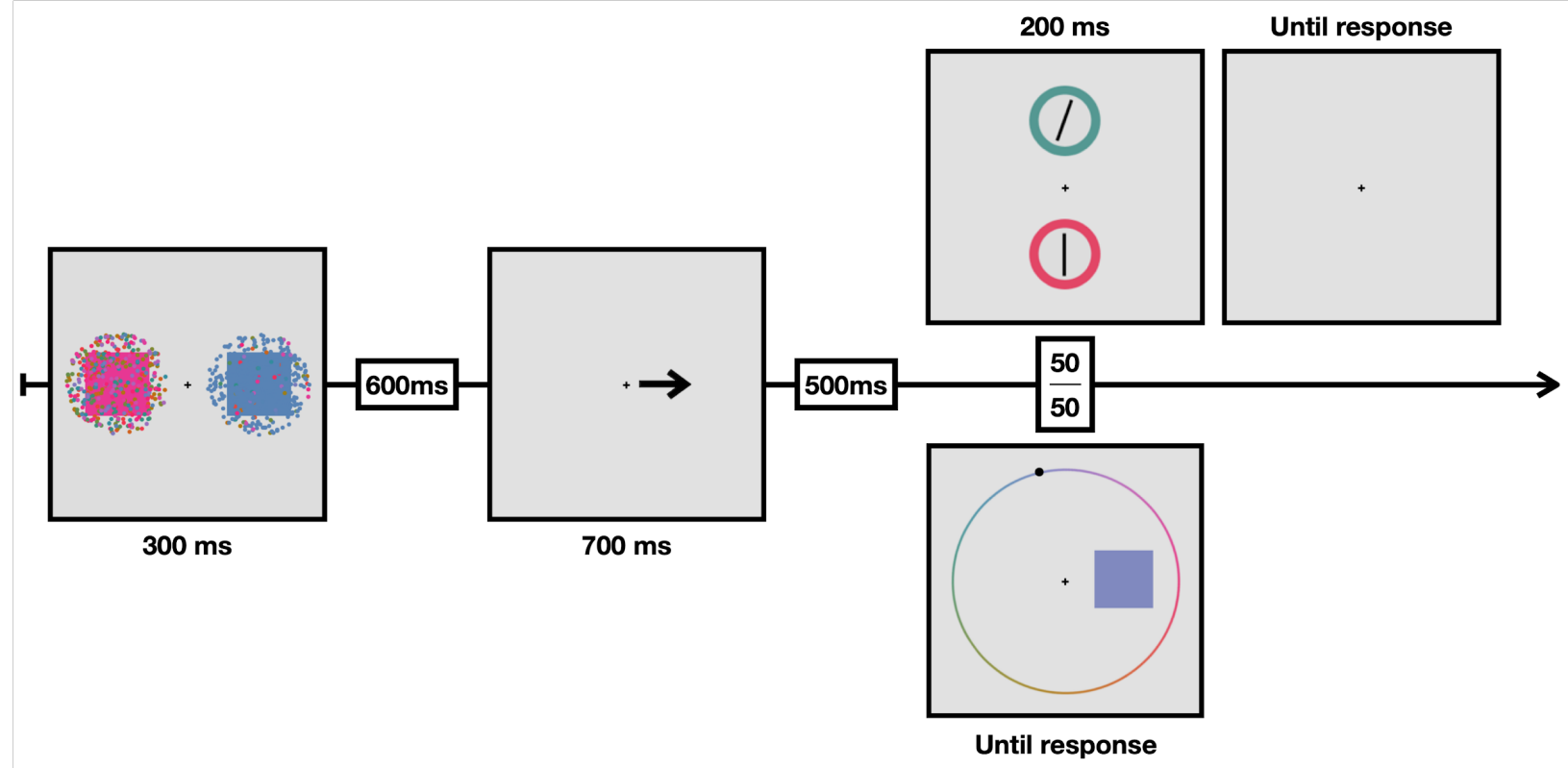

Figure 8. Experiment 5 Task design. Participants remembered two items: one high-noise (left) and one low-noise (right) item across a short delay. A retro-cue signifies which item will be tested on (forced report) memory trials ( $80 \%$ valid on memory trials; not predictive on search trials). On half of trials participants performed a search task and on the other half of trials had their memory randomly tested (see Methods for more details).

\section{Methods}

\section{Participants}

Due to the global pandemic, in-lab data collection for this experiment stopped prematurely. We transitioned to an online study and, to make it more amenable to online testing, changed the length of the experiment, the sample size, and multiple aspects of the task itself (preliminary in-lab results mirror those found here). All participants were between 18 and 36 years old, reported normal or corrected-to-normal vision, and gave informed consent in accordance with the procedures approved by the Institutional Review Board at UC San Diego. 135 participants (72 females) from UC San Diego took part in this online study in exchange for course credit. Exclusion criteria were identical to Experiment 1 and resulted in the removal of 35 participants, giving a final sample size of 100 participants. 
Memory fidelity drives guidance

\section{Stimuli}

Participants performed the experiment on their home computers on a monitor that was at least $800 \times 800$ pixels to ensure the entire display was visible for the duration of the experiment. Similar to Experiment 2, two colored squares (90x90 pixels) were placed either side of fixation (300 pixels apart; centrally positioned 150 pixels to the left or right of fixation). Memory items were drawn from the same color space used in previous experiments and the color value for memory items were roughly $90^{\circ}$ apart. Similar to Experiment 2, a circular cloud (50-pixel radius) of perceptual noise was superimposed over the memory colors. One memory item was occluded by 360 uniquely colored dots (4-pixel radius each; designated High-Noise item) and the other memory item was occluded by 60 uniquely colored dots (evenly spaced by $16^{\circ}$ across the color wheel) and 300 dots that matched the color of the memory item (designated Low-Noise item; see Figure 10). The search display was identical to previous experiments except where noted: search items were 150 pixels above or below fixation and contained either a straight line (distractor; 4 pixels wide and 55 pixels tall) or the target line which was identical to the distractor line except that it was titled by $30^{\circ}$ either clockwise or counter-clockwise. The search display briefly appeared for $200 \mathrm{~ms}$ before disappearing and showing only the fixation cross.

\section{Procedure}

Participants performed a total of 320 trials that were evenly split between search trials and memory probe trials. On each trial two memory items were presented for $300 \mathrm{~ms}$; one memory item was designated high-noise and the other item was designated low-noise. The location of each item varied randomly across trials. Participants maintained these items over a $600 \mathrm{~ms}$ delay prior to the presentation of a retro-cue $(700 \mathrm{~ms})$ which signified which item would be tested for memory with $80 \%$ validity (non-predictive of search; $50 \%$ like to be present on 
search trials). The retro-cue was aimed at the high- or low-noise item evenly (160 trials each).

After a final 500 ms delay the search or memory probe display was presented.

The search display was the same as in previous experiments (note: the search task was only displayed for 200ms). Participants used the left or right arrow key on the keyboard to report the target orientation (counter-clockwise and clockwise respectively). Immediately after a keypress, participants received feedback on their performance. On memory probe trials participants saw the memory probe display (see Figure 8) which informed them to report the cued or uncued memory item. Participants interacted with this task exactly as before; using the mouse to move around the continuous color wheel until the reported color matched their memory as closely as possible. Feedback was provided after a response was made.

A
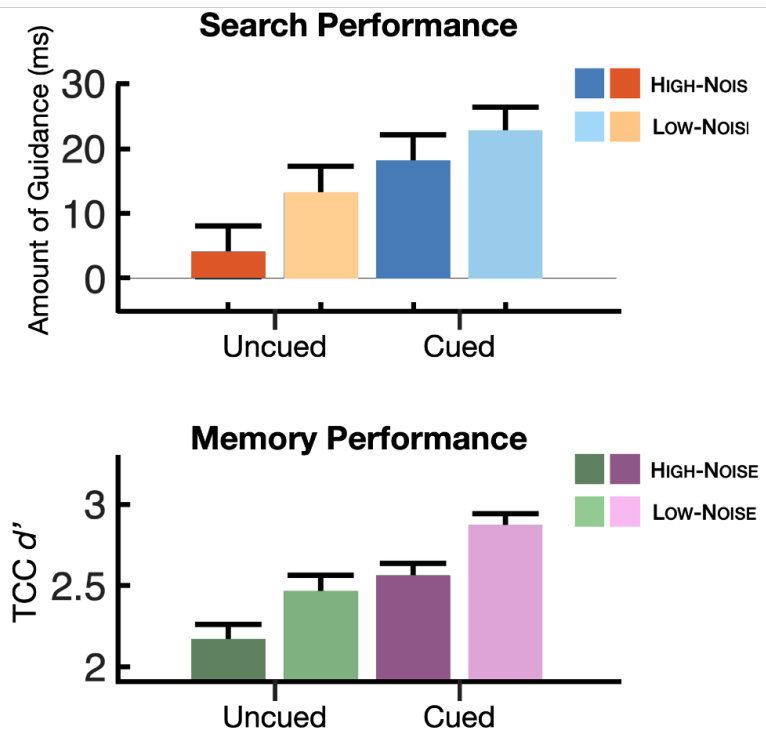

B
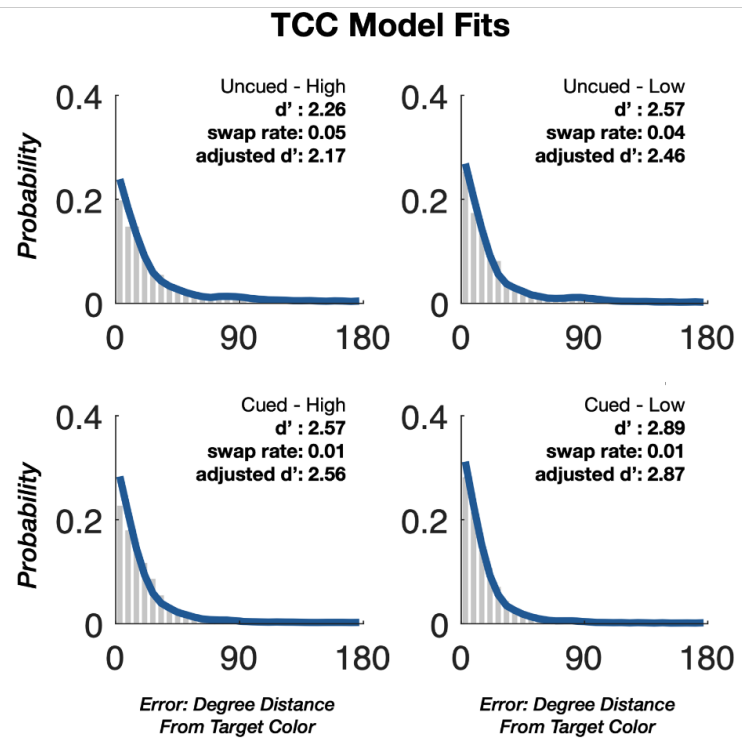

Figure 9. Experiment 3 results A) Top-Left: Search effect for each of the four noise and cue conditions. Even for items putatively "within the focus of attention" by a directional cue (blue bars) more precise memory representations (low noise) resulted in a larger search effect compared to less precise representations (high noise). When an item was un-cued, i.e. "outside of the focus of attention" (a 'template status' had been given to the other item), guidance was similarly dependent on the representational fidelity of the item. That is, low-noise uncued items exerted robust guidance over attention, while high-noise (poorly represented) items did not. In short, the search effect followed memory performance regardless of whether an item was cued 
or not. Bottom-Left: Memory performance (adjusted d') was highest for cued items that were encoded with less perceptual noise (low-noise) and worst for uncued items that were encoded with more perceptual noise (high-noise). B) Corresponding error histograms and TCC model fits for all conditions.

\section{Results \& Discussion}

In Experiment 3 we manipulated the representational fidelity of both items in working memory on the same trial and found (1) that the amount of attentional guidance follows the same pattern as the memory fidelity of these items and (2) that uncued items-unattended items with no template status-guide attention as long as they are well represented (Figure 9). For memory performance, we found significant main effects of perceptual noise $(F(1,99)=$ 52.92, $p<0.0001)$ and cue condition $(F(1,99)=43.7, p<0.0001)$ and no interaction $(F(1,99)=$ $0.03, p=0.86)$. For search, we found significant main effects of cue validity $(F(1,99)=8.56, p<$ $0.001)$ and noise manipulation $(F(1,99)=4.17, p<0.05)$, and no interaction $(F(1,99)=0.45, p>$ 0.05).

In planned t-tests we found a significant search effect for cued items (i.e., a template item); irrespective of whether the memory item was subjected to high-noise $(t(99)=3.92, p<$ $0.001)$ or low-noise $(t(99)=5.28, p<0.001)$. Of particular interest, for uncued items (those without any template status) we found a significant search effect for low-noise items (t(99) = 3.16, $p<0.01)$ but not for high noise items $\left(t(99)=0.57, p>0.05 ; B F_{01}=7.71\right)$. These search results demonstrate that well represented items guide attention, that poorly represented items do not, and, critically, that an uncued item without a template status guides attention when it is well represented.

While these results strongly argue against an attentional template account, it is plausible that when a poorly represented item is cued (high-noise-cued trials), participants do not focally attend to it and instead focus on the less noisy, uncued item. If true, we would expect a higher swap rate when the high-noise item was cued compared to when the low-noise item was cued 
since, if participants were internally attending to the wrong item, they would be more likely to report that item at test. However, we find no evidence to support this proposition as swap rate was effectively non-existent, and very similar between high-noise and low-noise conditions (0.005 vs 0.007 , respectively, $t(99)=0.69, p=0.49$ ) and we found compelling evidence to support this null finding $\left(B F_{01}=7.17\right)$. These results lead us to conclude that participants maintained cued items as an attentional template (i.e., did not swap to better represented items) and that the search effect on the low-noise-uncued condition is from an item without a template status.

These results fit well with the results from Experiments 1-2 in suggesting that memory fidelity underlies the amount of observable guidance. They are also consistent with the idea that retro-cues are simply one way-a particularly effective way-among many others, to boost the representational fidelity of an item, which has downstream effects on guidance. Furthermore, these results are consistent with our representational fidelity account where any and all memory items guide attention insofar as they are well-represented. They are also, however, inconsistent with an attentional template account where only centrally focused item(s) can guide attention.

\section{Explaining guidance effects across all experiments}

Our proposed account makes a strong prediction, not just within experiments but also across experiments: memory fidelity is sufficient to explain attentional guidance, with no other factors needed (e.g., with all other factors exerting influence purely through their effect on representational fidelity). Our data are qualitatively consistent with this prediction: Experiment 1 suggests that within a trial, items that are best represented are most responsible for search effects, and Experiments 2 and 3 show that average memory performance predicts the average magnitude of the visual search effects, such that stronger memory representations were related 
to stronger guidance effects across trials. These conclusions include across-trial comparisons of memory performance where there was no possibility of re-encoding the item during search and thus having the search display influence memory or vice versa (preliminary Exp 1A-2A and Exp 3).

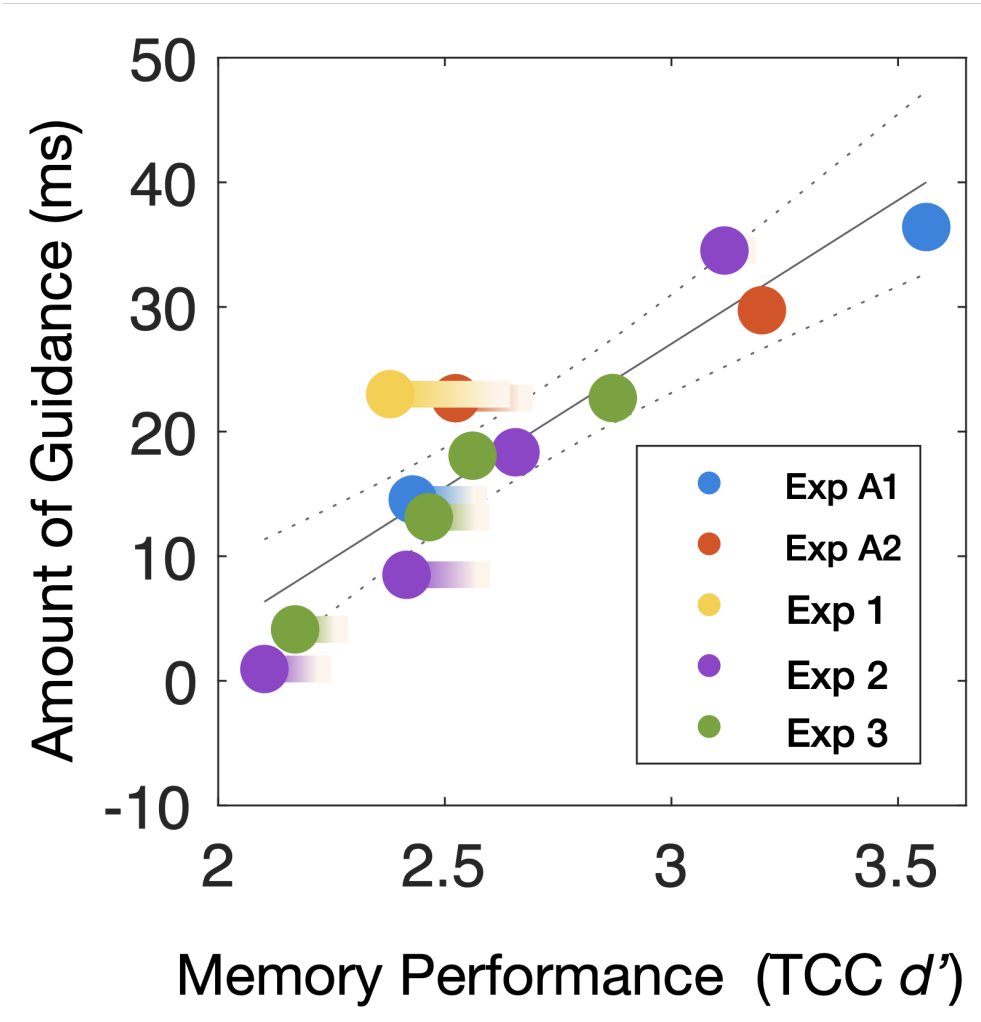

Figure 10. Each experiment is plotted as a unique color; multiple dots represent unique conditions. Dots correspond to memory performance: represented as the adjusted d' (d' for the probed memory item; a conservative measure which assumes participants have no familiarity for the probed item and decided to report the other one), since performance for the probed item is most relevant when asking about how memory relates to the search effect. Each dot has a 
corresponding colored line which represents the total possible range of memory strength: if participants maintained perfect memory representation for both items and simply reported the wrong location, d' would be at the far right of each line. In general, the amount of guidance increases with memory performance within and across experiments.

To quantitatively test the prediction that representational fidelity can explain the amount of attentional guidance, we correlated average memory performance with the average guidance effect across conditions from all of our experiments-including two supplementary experiments in which we varied set size (1 vs. 2, see Appendix). We included only forced report trials as they reflect the true underlying memory fidelity whereas free report trials overestimate the underlying fidelity of items as they are biased towards items that happen to have the highest representational fidelity on a particular trial. We submitted memory performance (TCC adjusted d) and the amount of guidance (distractor-match minus target-match RT) to a simple linear regression model and found that memory performance was highly predictive of the guidance effect across experiments (Pearson's $r=0.9, \mathrm{R}^{2}=0.82, p<0.0001$ ). For this analysis, we used adjusted $d$ ' which assumes that individuals have no familiarity for the probed color when they report a color from the incorrect location; an assumption that results in conservative estimates of memory. Instead, it is likely that even when location information is lost, resulting in a swap, this deterioration does not lead to a total loss of information about the color (i.e., zero familiarity). Thus, we also plot the d'values that presume memory strength is just as strong on swap trials to show the total possible range (the horizontal line connected to each point), and, as can be seen in Figure 10, this does not alter the nature of the relationship. This correlational analysis, with a clear increase to an item's ability to guide attention as that item is better represented, thus provides support for the hypothesis that the strength of memories is a critical factor in determining guidance effects.

In addition, as seen in Experiments 2-3, this analysis suggests that very strong memories are needed to guide search: The intercept of zero memory guidance is predicted to 
appear at approximately a $d^{\prime}$ ' of 2 , which is still a strong and extremely accurate memory. This may explain why natural variation between items in representational fidelity is sufficient to cause guidance to be largely driven by a single item, even at set size 2: even slightly deleterious noise is likely to remove the ability of an item to guide attention.
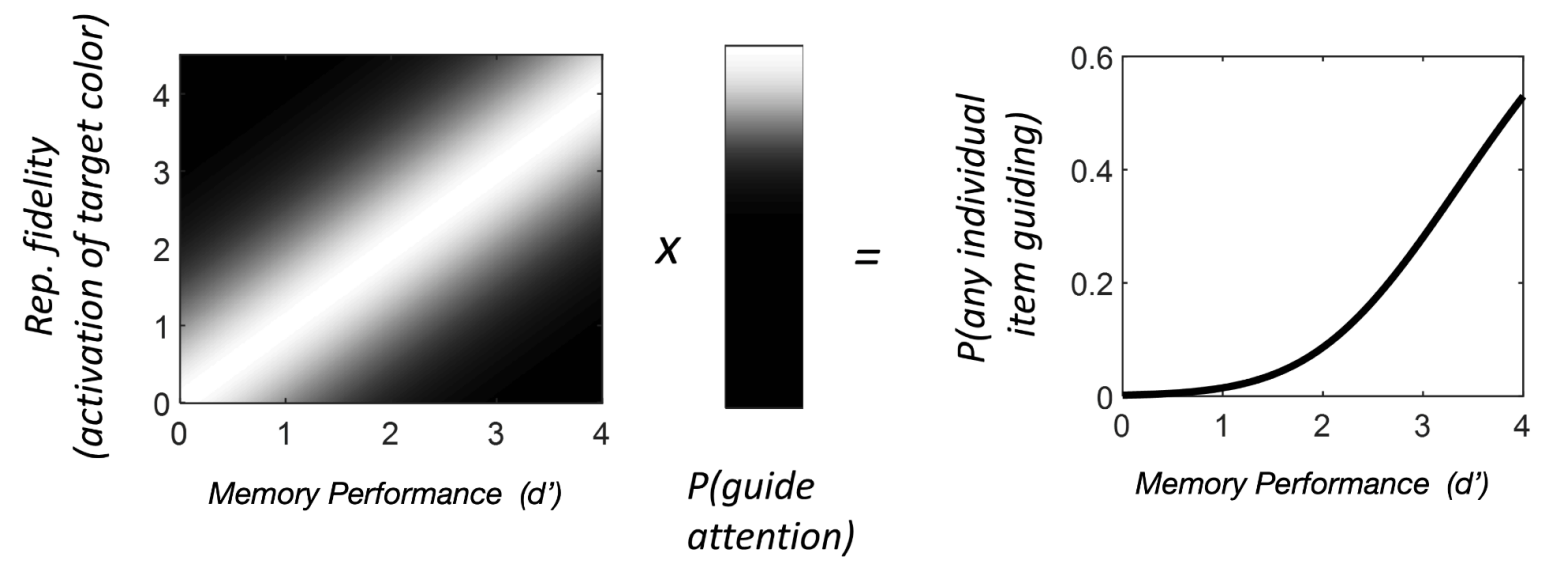

Figure 11. Our final proposed model has two premises: Items vary in representational fidelity, even from the same display, because of independent noise; and only strong items guide attention. Left: Even for items encoded with the same memory strength, the ultimate representational fidelity varies between items. In the TCC model we use to measure memory, this variation between items is a normal distribution with $S D=1$, consistent with signal detection theory. Middle: Combined with the variation in precision between items is the fact that only strong items guide attention. The plot shows one possible instantiation of this, with the likelihood of attention being guided by an item based on the item's representational fidelity (here, $\Phi$ (Mean $=3.5, \mathrm{SD}=0.5)$ ). Right: The result of this is that guidance occurs only when the items tend, on average, to be strong, matching Figure 10's real data.

In summary, then, the model we propose can be instantiated as shown in Figure 11. This model has two parts: (1) First, memories, even at the same set size, vary independently in representational fidelity (Fougnie et al., 2012; Schurgin et al. 2020; Panichello, DePasquale, Pillow, \& Buschman, 2018; Wilken \& Ma, 2004). In the TCC model we use throughout, this is implemented via signal detection theory. Thus, even when both items at set size 2 are encoded with $d^{\prime}=2.0$, there is variation in the ultimate representational fidelity of these items, in part, because they accumulate independent noise (Fig. 11, left). (2) Second, our data strongly 
suggest that only items with high representational fidelity guide attention in a robust and reliable way. Figure 11 (middle) instantiates one particular version of this, where strong representational fidelity is required to guide attention.

Together, these two premises are consistent with the patterns of data we observe and that are observed in the literature. The particular numbers from Figure 11's instantiation are not necessarily fixed - they depend on various assumptions about what it means for only strong memories to guide, and how guidance might affect reaction time. However, the patterns remain the same regardless of these parameters. Such a model matches Figure 10 in terms of guidance as a function of average memory strength $\left(d^{\prime}\right)$. This model also predicts other effects we observe, like the heterogeneity between items even on the same trial. For example, at set size 2 , with $d^{\prime}=2.5$, the model predicts that any given item has a $30 \%$ chance of causing guidance. However, since the noise for each item is independent, this implies that the chance both would guide attention is only $9 \%\left(30 \%{ }^{\star} 30 \%\right)$, suggesting most trials will have only one item guide attention in a meaningful way.

\section{General Discussion}

Recent work has shown that attention can be biased towards items that match the contents of working memory. Using hybrid visual working memory and visual search paradigms, several studies have shown robust guidance when a single working memory item is maintained (Olivers et al., 2006; Soto et al., 2005, 2008; Soto \& Humphreys, 2007, 2008) though the results are somewhat mixed when multiple working memory items are held in mind (Beck \& Vickery, 2019; Chen \& Du, 2017; Fan et al., 2019; Frătescu et al., 2019 Hollingworth \& Beck, 2016; Hollingworth \& Hwang, 2013; Houtkamp \& Roelfsema, 2006; van Moorselaar et al., 2014; B. Zhang et al., 2018). The discussion of whether multiple items can guide attention is often focused on the number of items that can achieve a privileged template status with little focus on 
the representational fidelity of the memoranda. Here, in contrast, we carefully assessed the representational fidelity of working memory items, demonstrating a straightforward relationship between the representational fidelity of memories and attentional guidance that exists independently of an item's template status. In particular, we found that both within-trial and across-trial variation in representational fidelity predicted attentional guidance and did so without needing any other predictors (like a special privileged state). These findings strongly suggest that the degree to which an item accurately represents the originally encoded item (ie, the representational fidelity) determines whether-and how effectively-an item guides attention.

In particular, we show that when two items are maintained in visual working memory, one of the items tends to have greater representational fidelity than the other item, suggesting natural and inherent variation between memories; that the more accurate representation primarily drives the observed guidance effect while a poorly represented item exerts little to no influence over attention (Experiment 1); that the observed variation in memory reports between items is predicted by basic signal detection theories of memory (Simulation, Figure 5B); that, across-trial variation in the quality of memory representations predicts the size of search effects (Experiment 2); and, finally, that attention is guided by well represented items irrespective of achieving any purported template status (Experiment 3). Importantly, Experiment 3, along with the correlation across experiments (Figure 10), suggests that multiple working memory items are each capable of guiding attention, as long as that item is maintained with sufficiently high representational fidelity (although this may be a rare occurrence in typical paradigms; see Figure 11). While these latter results support a representational fidelity account they are also in stark contrast with fundamental assumptions of the attentional template account.

The proposed representational fidelity framework speaks to two important issues in the literature of memory driven attentional guidance as well as to working memory and attention literature more broadly. First, to the question of whether one, or many working memory 
representations guide attention. Our data indicate that only an extremely strong and high-fidelity memory representation can guide visual search effectively, something that rarely occurs for more than one item at a time (e.g., Figure 10). To be clear, we do not suggest that multiple items could never guide attention simultaneously (as evidenced by Experiment 3), instead, the data simply suggests that all of the simultaneously maintained memories are unlikely to be sufficiently well-represented to each exert strong guidance over attention.

Second, the present results elucidate the mechanisms underlying attentional guidance and explain why attentional guidance is often driven by a single item. Importantly, and different from previous accounts, our data suggest that natural variation in the representational fidelity between items is sufficient to explain the extent to which an item will guide attention on a particular trial, with no special focus of attention or similar state-based accounts of working memory being necessary (Experiments 2-3 and the Simulation). Under this account, retro-cues are simply one way to improve memory fidelity, but a similar boost in memory-and a corresponding boost in attentional guidance-can also be accomplished differently, as we show in Exp. 2 and 3.

\section{The importance of representational fidelity}

The importance of strong memories and their inherent connection to an item's ability to guide attention has been acknowledged in the literature for many years. Unfortunately, however, this recognition has rarely translated into precise measurements of representational fidelity of individual items. For example, in an elegant set of experiments, Olivers, Meijer, and Theeuwes (2006) found no search effect when participants knew that the final memory test would be relatively easy (ie, red vs green) but found a substantial search effect when participants knew that the memory test would be difficult (eg, two subtle variations of red). While the goal of this manipulation was to assess the differences between verbally and visually maintained 
representations, the more difficult memory condition had the likely effect of producing higher representational fidelity for remembered items.

More importantly, much of the literature on attentional guidance does not use tasks that allow for direct measurement of memory strength for the relevant features at all. So, despite designing tasks that would encourage participants to maintain a highly precise representation, the memory probe itself cannot lead to an accurate estimate of representational fidelity. This is because tasks were used in which performance depends on memory for features that are not relevant for guidance (Chen \& Du, 2017; King \& McNamara, 2020), or 2-AFC probes where the foil items are extremely similar to the target color ( Hollingworth \& Beck, 2016; van Moorselaar et al., 2014 Exps 1-2 and 4). These manipulations make accurate assessments of memory strength impossible because they decrease estimated performance without changing the underlying memory signal, as shown by Schurgin, Wixted, and Brady (2020). Furthermore, these manipulations are often combined with measures of memory that are not independent of response criterion, for example by averaging percent correct in a change detection task (e.g., B. Zhang et al., 2011; Frătescu et al., 2019, Dude et al., 2019) - a method which embraces a highthreshold model of responses, even though memory appears graded in nearly all studies, including in working memory studies (see Robinson, Benjamin, Irwin, 2020).

In the case where studies use foils that are extremely similar to the target (e.g., Hollingworth \& Beck, 2016; Olivers et al. 2006; van Moorselaar et al. 2014; Frătescu et al., 2019) their performance estimates effectively compress the performance scale-very strong memories are needed to get a $d^{\prime}$ 'above 0 , and a $d^{\prime}$ ' of 0.5 in such a task might correspond to a $d$ ' of 3 or more in a task like ours or in a 2-AFC task with more distinct foils (Schurgin et al., 2020). This is to say that memory estimates from previous studies are not directly comparable for many reasons. While Schurgin et al. (2020) demonstrate that 2-AFC tasks with maximally distinct foils effectively measure the same underlying memory strength as comparisons between more 
confusible colors or as continuous report, the nature of the seemingly low performance in some attentional guidance studies (eg, 65\% accuracy) leads many of these researchers to interpret memory fidelity as all-or-none (i.e., precise or not precise), potentially obscuring the strong relationship between memory fidelity and guidance.

Some attempts have been made to look at memory fidelity and guidance using more exact measures. However, even findings which more accurately estimate memory have sometimes claimed no relationship between memory strength and guidance by looking at individual trial errors (Hollingworth \& Hwang, 2013). However, no models of memory support a direct linear relationship between error on a single trial and the underlying representational fidelity of memory, especially not models where responses are inherently stochastic (e.g., Schurgin et al. 2020; Bays, 2015). For example, sampling from the TCC model shows that even if $d^{\prime}$ was $100 \%$ perfectly predictive of guidance, the maximum correlation observed in such an analysis would be $r=0.09$ (since a given $d^{\prime}$ 'can result in any error, with only a slight change in their proportions). The null result observed in this study and studies like it are thus not informative for the central issue of whether memory fidelity might underlie variations in guidance.

By contrast, in the current work we put a strong emphasis on accurately measuring memory quality for items and directly relate these measures to the guidance effects both within trials, across trials, and across experiments. This allows us to make clear predictions about which items guide attention and even allows us to quantify the representational fidelity that is needed for an item to guide while also determining how likely it is that more than one item exerts an effect during visual search (Figure 11). Concurrent recent findings have also demonstrated how accurately estimating memory strength elucidates its importance to the magnitude of the guidance effect (Kerzel, 2019; Kerzel \& Witzel, 2019). For example, Kerzel and Witzel (2019) find that a secondary working memory item does not guide attention and that 
this is not due to the lack of a template status, but is simply due to that item being maintained with less representational fidelity than the guiding item. Similarly, Kerzel (2019) suggests that the number of guiding items is fewer than the overall capacity of working memory because the precision of guiding items must be extremely precise, not necessarily because a narrow (single item) attention template drives the effect. In the future, to further understand the role of representational fidelity in attentional guidance, it would be useful for those studying attentional guidance to use memory measures that precisely and accurately assess memory strength, and ideally measures that would allow for a comparison between studies. This could be achieved by using 2-AFC with maximally different foils at test, which would provide a measure of the upper bound of memory in these tasks (Brady \& Störmer, 2020), or by reporting either TCC $d$ ' (Schurgin et al. 2020), or the circular standard deviation when using continuous report.

\section{On the number of items that guide attention}

Many studies have found guidance effects for one and two item working memory loads, including our supplementary experiments (see Appendix; Olivers et al., 2006; Soto et al., 2005, 2008; Soto \& Humphreys, 2007, 2008; Beck \& Hollingworth, 2017; Beck et al., 2012;

Hollingworth \& Beck, 2016; Kerzel \& Witzel, 2019; Soto \& Humphreys, 2008; B. Zhang et al., 2011). Such multiple-item guidance effects can always be explained in two ways: (1) both items equally guided attention, or (2) one working memory item is primarily responsible for driving the multiple item effect (Downing \& Dodds, 2004; Beck \& Vickery, 2019; van Moorselaar et al., 2014; Olivers et al., 2006; Olivers et al., 2011; Zhou, Lorist, \& Mathôt, 2020). Historically, dissociating between these two interpretations has been extremely difficult, as they mimic each other when averaging across trials. The representational fidelity account, described here, offers a new view on this question and has the potential to explain which cases would result in the guidance of attention by only a single item or multiple working memory items. 
In many cases, the variation in fidelity across remembered items that we observe-and find to be extremely important for guidance-seems to undermine the strongest claims of two item guidance. For example, Hollingworth and Beck (2016) had participants maintain one or two working memory items while they searched for a single target among eight distractors. Using search displays with two distractors that could match one (match-1), both (match-2), or none (match-0) of the memory items, they showed that attention was guided on both match-1 and match-2 trials and found a greater effect when both items appeared in the search display. While these results appear generally consistent with multiple items influencing attention, an alternative explanation is that because the best represented item was more likely to be present on the search display in the match-2 condition, a more consistent, and thus more robust, guidance effect was found. Specifically, on match-1 trials the best represented item would be expected to be present on $50 \%$ of trials, and on match- 2 trials, the best represented item would be present on $100 \%$ of trials, which would generate a greater, and more reliable search effect in the match2 condition on average, even if only a single item was guiding attention (a similar logic applies to a replication of this original study by Frătescu et al., (2019), and other studies with a similar design, e.g., Fan et al., 2019; Zhou, Lorist, \& Mathôt, 2020). Thus, in these types of tasks, the presence of random variation in memory fidelity between items, combined with only strong items guiding attention, potentially makes it difficult to know with confidence how much guidance is genuinely arising from the second item.

Of course, our account does not suggest that only one item is necessarily responsible for guiding attention: on some trials, when both items are represented extremely well, we predict that both items could exert at least some observable guidance. And, while this situation is unlikely to occur when participants are asked to remember colors that are randomly drawn from 360 unique colors, there are manipulations that could modulate memory strength to produce such an effect. A possible example of this principle is provided by a recent study by Chen and 
Du (2017) where they investigated whether two memory items could guide attention by combining two critical features from previous studies: a match-2 condition (Hollingworth \& Beck, 2016) and a shape singleton search task (van Moorselaar et al., 2014). Across a convincing set of experiments, they provided data which suggested that multiple items can exert roughly equal guidance over attention. When participants remembered two items and were randomly presented with one of those items as a distractor (match-1), they found that attentional guidance was roughly equal when either memory item appeared. On trials where both items appeared as distractors (match-2), the attentional guidance effect (measured by their memory-driven capture index) was roughly double that of the match-1 condition and the guidance effect on match-2 trials was greater than when a single, cued memory item appeared in the search display. Thus, these findings appear to demonstrate that two items are capable of exerting roughly equal, additive guidance over attention. This finding is well explained by our representational fidelity account as it likely originates from the extremely well represented nature of the memory items: both items appeared to be represented with roughly equal precision (as measured by their 8AFC task; see their Table 1), and memories were likely extremely strong because participants were shown two- of only four possible-unique colors ${ }^{4}$ for long encoding time $(1000 \mathrm{~ms})$ on every trial; likely supporting the creation of strong and less noisy memories.

Across our experiments, participants were shown a much larger stimulus set than is common in this literature (e.g., randomly selected colors from a continuous color wheel; 360 unique items). When two items were maintained, we found that memory strength for one of these items is substantially greater than the other and studies which have been taken to support

\footnotetext{
${ }^{4}$ Note that the authors did not intend to use a small stimulus set and attempted to increase the reliance on working memory by including three textures that were superimposed over the colored disks. This resulted in a total of 12 items to-be-remembered. However, in such circumstances, color memory-the feature responsible for the guidance in this task-is generally almost always independent of other features (e.g., Fougnie \& Alvarez, 2011), and so color memory was only tasked with remembering a total of 4 unique items.
} 
a multiple-item template account have also shown this pattern for remembered items. For example, B. Zhang et al. (2018) used a paradigm similar to ours while also tracking eye movements: Participants remembered two colors (sampled from 180 unique values) while they performed a simple, two-item search task. Participants were cued as to which item would be tested first, and on half of the trials, participants reported their memory strength on a continuous color wheel instead of searching for a target among a single distractor. While response time data from search trials was roughly equal for both memory items (cued and uncued) the authors measured first fixations as a more sensitive measure of a memory item's control over attention. While they conclude that multiple items guide attention, their data show that the proportion of first fixations tracked the reported memory strength of each item. That is, the cued item was fixated more often than the uncued item and the cued item was also maintained with greater fidelity. Their findings suggest that less well-represented items can interact with attention, but that they do so less efficiently and in direct relation to how well they are represented in memory. These results are similar to our data from Experiment 3 and, therefore, are in line with a representational fidelity account, which postulates that in principle any and all items can guide attention, but that the amount of guidance is determined by the underlying representational fidelity which can be modulated by attentional cues.

\section{Memory fidelity and the focus of attention}

We show that differential memory performance between items (as indexed by different performance in forced vs. free report) arises in almost every situation (Exp. 1, and Supplementary Exp. ), consistent with several other studies (Adam, Vogel, \& Awh, 2017; Bays, Catalao, \& Husain, 2009; Brady \& Alvarez, 2015; Fougnie et al., 2012; W. Zhang \& Luck, 2008; Brady \& Alvarez, 2015; Fougnie et al., 2012). Why do memories vary in their representational fidelity, and how does this variation relate to attentional guidance? According to an attentional 
template account, one item is selected among other items, thereby getting a boost in memory performance while also gaining the ability to interact with attention. Why-and how-items are selected by attention and granted priority is often not specified, and it is an open question as to how this internal spotlight of attention acts upon memory representations especially with respect to when a representation is selected without top down control or explicit instruction. The majority of previous work has used pre-and post-cues to manipulate this focus of attention and has found that attended items guide attention more effectively, consistent with the focus-of-attention account (Olivers et al., 2011; van Moorselaar et al., 2014). However, in all of these studies the putative focus of attention and the quality of the memory representations were varied at the same time, because attended working memory items also resulted in better fidelity memories. Attentional cues changing memory fidelity could arise from a variety of sources, however, for example by devoting a greater proportion of resources to this item, or because it accumulates less noise as a result of being protected from interference (Bays \& Taylor, 2018; Gazzaley \& Nobre, 2012; Griffin \& Nobre, 2003; Souza \& Oberauer, 2016). Thus, it is possible that the focus of attention effects are simply effects of changes in memory quality.

Given that attentional priority typically co-varies with improvements in memory performance, it is difficult to disambiguate between these two accounts. In Experiments 2 and 3 , we designed a task that, for the first time, teased apart effects of attention and memory quality on attentional guidance. By adding perceptual noise at encoding, we manipulated the representational fidelity of each item. This memory manipulation was independent of any attentional cues, and by using a post-cue after a short delay we manipulated priority, independently of the encoded precision. Our results showed that the post-cue enhanced memory performance-as expected-and at the same time produced robust attentional guidance. Critically, however, we found that perceptual noise at encoding-which modulated 
memory quality but not attention-also influenced the guidance effect of attended items such that noisier items showed a smaller guidance effect, even when cued ('placed within the focus of attention', by such accounts; see Figure 10). Experiment 3 in particular revealed that items that are not cued (i.e., outside the focus of attention and with no template status, by such accounts) also showed guidance effects as long as their memory was strong enough, as predicted by the representational fidelity account but wholly incompatible with an attentional template account.

Thus, we argue a straightforward and parsimonious explanation for differences in working memory guidance is that they arise from variations in the representational fidelity of items both within and across trials, and not because any item achieves a special status by being placed within a focus of attention. Rather, a framework in which representations vary in fidelity due mostly to independent noise but also modulated by display characteristics (e.g., Brady \& Alvarez, 2015), cues, uneven resource allocation (Bays \& Taylor, 2018), and more-but that does not require additional assumptions about differential states or templates to support item representations - can fully account for the present data, and a large set of data in the attentional guidance literature in general. This interpretation is also in line with signal detection models like the TCC memory model we use to fit our data, which predict natural variation in memory due to independent noise in the item representations (Schurgin et al., 2020). In fact, our simulations showed that the amount of variation in memory performance we find in our data is predicted by the natural stochasticity of signal detection theory, as implemented by TCC, with no need of any other explanations (like a focus of attention).

Because previous studies did not precisely measure differences in the representational fidelity between items and how that relates to guidance effects, evidence for one item guiding attention was taken as evidence of a fundamentally distinct state of certain items in memory (and indeed this concept has been invoked to explain differential precision as well; see 
Oberauer \& Lin, 2017). However, if items differ in how well they reflect the initially encoded item-due to independently accumulated noise during the delay period-as they appear to do in nearly all working memory studies (Adam et al., 2017; Brady \& Alvarez, 2015; Fougnie et al., 2012) and as we have shown here (Exp. A2 and Exp. 1), then it may be that poorly represented items do not bias attention simply because they are poorly represented. That is, as we show in Exp. A2, at set size 2, one item tends to be extremely well represented-just as well as the one item at set size $1-$ whereas the other item is much less precisely represented. If an item is poorly represented, it cannot, by nature, guide attention to the color that was previously encoded. Even when an item is cued, and putatively granted a privileged status, memory strength is a critical factor (Experiment 2-3) and the putative focus of attention cannot override the influence of representational fidelity (Experiments 2-3, see correlation Figure 10); with representational fidelity-and the effects of cues on such fidelity-instead seeming to be sufficient to explain guidance. Thus, our results do not provide evidence in favor of any attentional template account, even though they do show that in most cases one item primarily guides attention. Our data are largely consistent with a simpler view where attention is guided only to the extent that an item is well represented; with no added assumption of discreteness in memory states.

\section{Conclusions}

Selectively attending to relevant information in the environment is critical as we are subjected to more incoming sensory information than we could possibly process at once. Working memory allows us to maintain information no longer available to the senses for further processing, and it is imperative that these two systems interact successfully to navigate our environment. Here, we demonstrate that attention is biased towards objects that match the contents of working memory-even if task-irrelevant. Importantly, we show that working memory 
Memory fidelity drives guidance

representations tend to guide attention only insofar as they are well represented, and that differences in representational fidelity between items is a natural process predicted by signaldetection theory. These findings have important implications for our understanding of the fundamental structure and processes involved in working memory and attention. Our interpretation of these results is that memory representations bias attention to the extent that they are well represented; an interpretation that succinctly captures much of the data in the memory driven attentional guidance literature and does so without needing to invoke distinct states or special classes for working memory items. 


\section{Appendix}

Before running the core experiments that support our memory fidelity account of attentional guidance, we ran 2 studies that sought to replicate basic findings that were necessary prerequisites of our account but that were not novel. Because these experiments do not provide new information but simply serve as the building blocks of our core experiments, we have put them in this appendix to reduce the burden on readers of the manuscript. In particular, in Experiment $A 1$, we verify that our paradigm finds guidance with both 1 item and 2 items in working memory, and greater guidance from 1 item than 2. In Experiment A2, we verify that memories vary in representational fidelity in this paradigm, by including a free report memory condition where participants can report their strongest memory item, which we find results in better performance than forced report.

\section{Experiment A1: Visual search task with a one or two item working memory load}

Participants maintained either one or two colors in visual working memory and were asked to report a remembered color on $20 \%$ of trials using a continuous report color wheel. On the majority of trials $(80 \%)$, instead of reporting the memory color, participants performed a visual search task in which the working memory color was irrelevant. Participants were instructed to report the direction of a tilted line (clockwise vs. counterclockwise from vertical). Based on previous research (e.g., Soto, Heinke, Humphreys, \& Blanco, 2005), we expected response times in the visual search task to be faster when the target was encircled by a color that matched the working memory color relative to when the distractor was encircled in the working memory color, reflecting the guidance of attention by a single visual working memory item. The main question was whether we would also find a guidance effect when participants maintained two colors in working memory. 


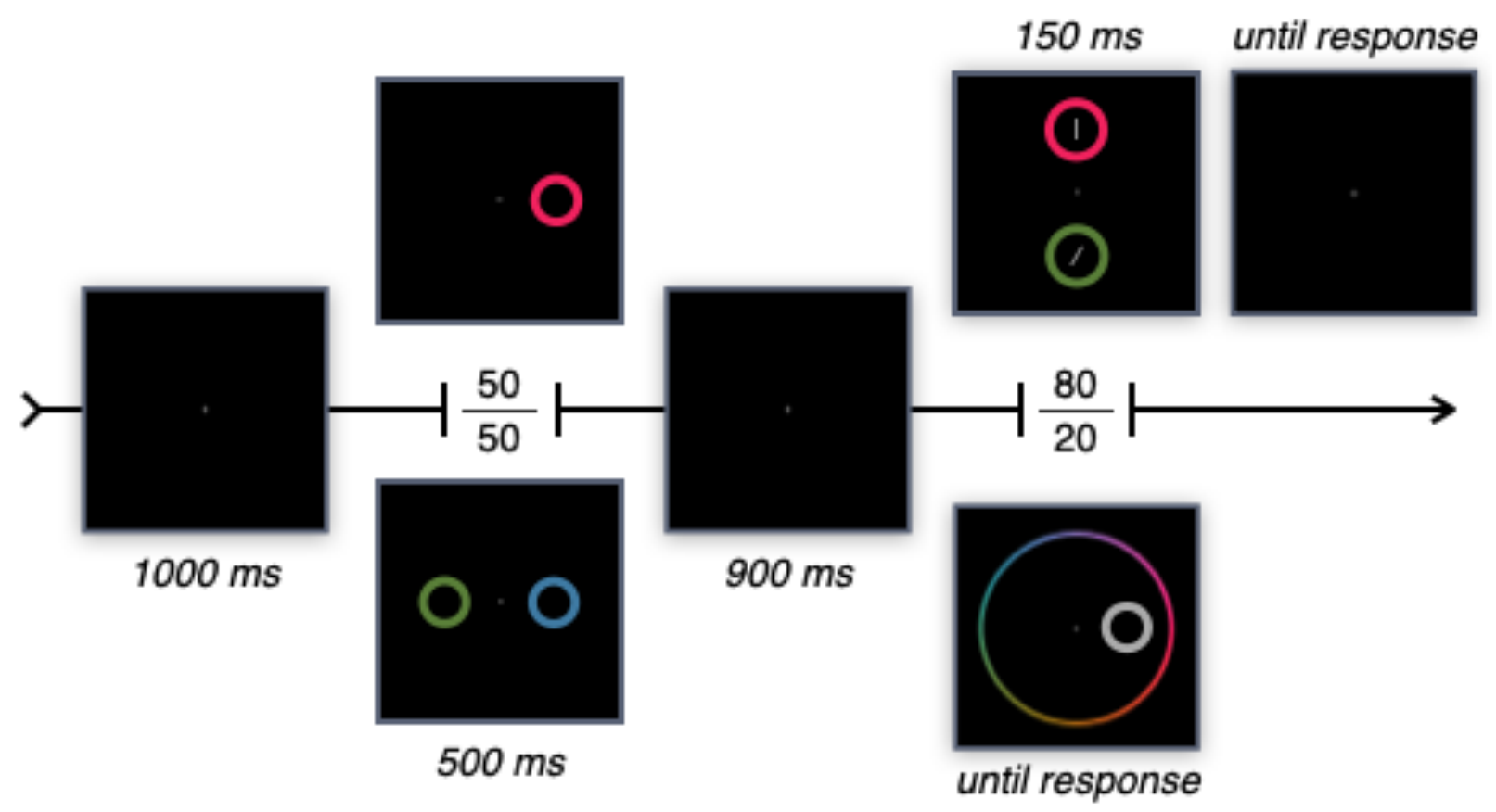

Figure A1. Experiment $A 1$ task design: Participants were asked to remember either one or two colors $(50 \% / 50 \%)$ on each trial over a short delay and then either performed a visual search task that required them to indicate the tilt direction of a target line (80\% of trials, top; target tilt is exaggerated compared to the experiment), or to report the color of one of the remembered items using continuous report (20\% of trials; bottom). In the visual search task, the memory color was not predictive of the target location or orientation. Experiment 1 was identical except for the addition of the free report memory condition (see Methods for details).

\section{Methods}

\section{Participants}

All participants were between 18 and 29 years old, reported normal or corrected-tonormal vision, and gave informed consent in accordance with the procedures approved by the Institutional Review Board at UC San Diego. Eighteen participants (13 females) from UC San Diego took part in this study in exchange for course credit. Data from two participants were excluded due to poor visual search performance ( $<50 \%$ accuracy). Additionally, one participant reported the incorrect memory item (i.e., the color of the item at the opposite location, i.e. "location swap") on more than $40 \%$ of all trials (more than 3 standard deviations from the group average). As such a high swap rate suggests that the participant failed to perform the task as 
instructed-reporting colors independently of the probed location-we excluded data from this participant as well, leaving 15 participants in the final sample. The results and interpretation hold with or without the removed subjects.

\section{Stimuli}

The experiment consisted of color stimuli presented on a black background. Stimuli were generated and presented using MATLAB and the Psychophysics Toolbox (Brainard,1997; Pelli, 1997). Memory items were colored rings that were $3^{\circ}$ visual angle in diameter, $0.3^{\circ}$ thick, and were centrally placed $4^{\circ}$ to the left or right of fixation. On every trial, the color of one memory item was randomly drawn from a uniformly spaced circle (radius $49^{\circ}$ ) cut out of the CIE $L^{*} a^{*} b$ space, centered at $(L=54, a=21.5, b=11.5)$ and when two memory items were present, the second color was selected to be $90^{\circ}$ away in color space from the first color. The search display consisted of a target line which was $0.3^{\circ}$ thick, $0.4^{\circ}$ long, tilted $0.06^{\circ}$ to the left or right of vertical, and placed $4^{\circ}$ above or below fixation and a single vertical distractor line that was placed at the opposite location (see Figure A1). The target and distractor lines were encircled in colored rings that matched the memory item properties except for their color. One of the colors matched one of the memory colors and the other color was chosen to be $180^{\circ}$ away from it in color space (at set size 2 , this was $90^{\circ}$ away from the other memory item). On the memory test display, one of the memory items was shown in gray (identical features to memory items) surrounded by a continuous color wheel which was $15^{\circ}$ in diameter, $0.3^{\circ}$ thick, and was centrally placed about fixation.

\section{Procedure}

Participants performed a total of 800 trials which were evenly divided between set size one and two. On each trial, one or two memory items were presented for $500 \mathrm{~ms}$ and participants were instructed to remember their color(s) as precisely as possible for a potential 
memory report task. After a 900ms delay, participants performed either the visual search or the memory task. On 640 trials (80\%) the search display was presented for $150 \mathrm{~ms}$ and participants reported whether the target line was tilted clockwise or counterclockwise from vertical by clicking the right or left mouse buttons, respectively. On 320 search trials (50\%), a memorymatched color encircled the target line (target-match) while a distractor color, $180^{\circ}$ away from the memory-matched color in color space, encircled the distractor line. On the remaining 320 search trials, a memory-matched color encircled the distractor line (distractor-match) while the distractor color encircled the target line. Thus, the memory color(s) never predicted which color the target line would be encircled by and was thus not useful for the search task. The location of the target and distractor line (top vs. bottom on the search display) was counterbalanced across the experiment. Feedback to respond more quickly was provided when responses exceeded $1200 \mathrm{~ms}$. On the remaining 160 trials (20\%) participants were presented with a memory test display that consisted of a continuous color wheel and a single gray test-item placed to the left or right of fixation. Here, participants were asked to use the mouse to find the color closest to the remembered color on the color wheel. The location of the test-item indicated which memory item should be reported (e.g., a test-item on the left probed the color of the memory item that was on the left at encoding), and which item was tested was counterbalanced across the experiment. Once the mouse was moved from the central fixation point the gray test-item changed color to match the color at the position of the mouse cursor. Once participants identified the color that matched the remembered color as precisely as possible on the color wheel, they locked their response by clicking the mouse button. Response error, defined as the difference in degrees between the provided response and the correct answer, was shown after every memory trial and participants were instructed to keep this error below $10^{\circ}$. Participants were instructed to prioritize speed without compromising accuracy for the search task and, for the memory task, were instructed to prioritize precision without compromising temporal 
efficiency. On set size two trials (i.e., two memory items were presented at encoding), one of the memory items was randomly selected to be either the memory-matched color on search trials or the tested item on memory trials. All trial types were randomly intermixed within each block (see Figure A1). At the end of each block (40 trials) participants were shown an average of their memory response error and visual search performance (RT and accuracy) for that block.

\section{Analysis}

The analysis for preliminary experiments 1-2 are identical to those described in the main text.
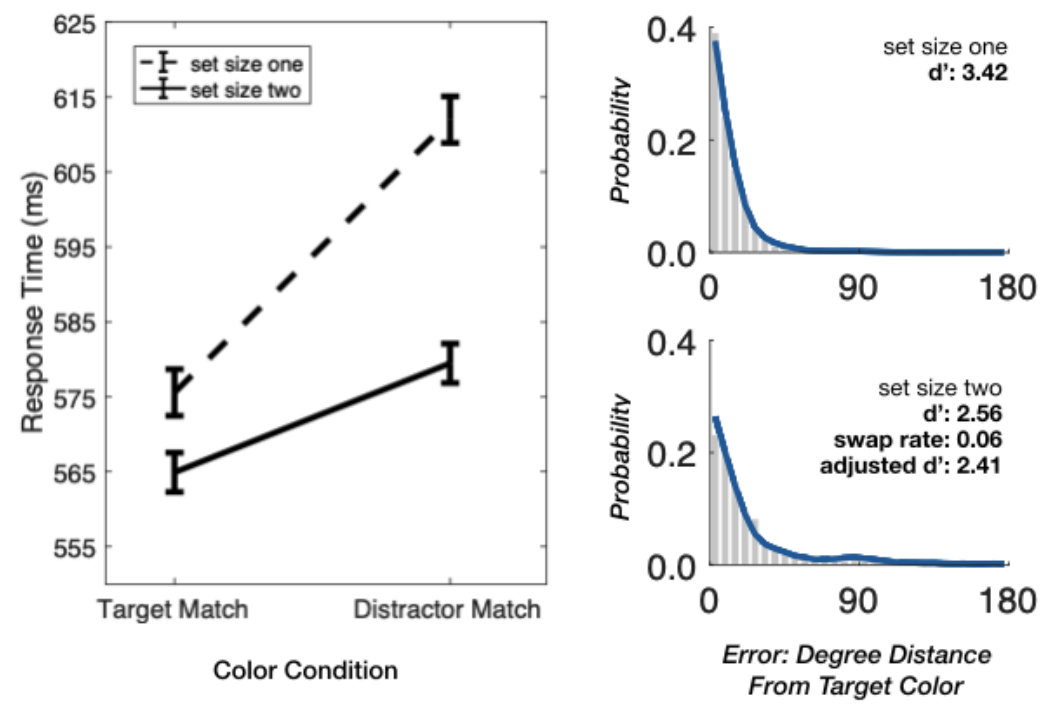

Figure A2. Experiment A1 results. Left: At both set size 1 and set size 2, search performance was faster when the target was encircled in a memory matched color (target match) compared to when it was encircled by a distractor color (distractor match; $180^{\circ}$ away from a memory item), showing attentional guidance by working memory items. Right: Memory performance on the continuous report task. Memory strength was superior (higher d') when a single working memory item was maintained (top) compared to two working memory items (bottom). The gray bars reflect histograms of participant's errors, and the blue lines are the model fits. On about 6\% of set size two trials, participants mistakenly reported the other (non-probed) memory item, signified by the slightly elevated responses at $90^{\circ}$ (location swaps). $d^{\prime}$ is the estimated memory strength for correct target reports only (ignoring swap trials), while adjusted d' reflects the memory strength when accounting for the likelihood that memory for the correct target was extremely weak when participants made location swaps. 


\section{Results}

The results of Experiment $A 1$ suggest that attention is guided towards items that match those being actively maintained in working memory, even when working memory is loaded beyond a single item (see Figure A2). We submitted search-color condition (target-match, distractor-match) and set size (set size one, set size two) to a $2 \times 2$ repeated measures analysis of variance (ANOVA). This analysis revealed two main effects and an interaction $(F(1,14)=$ 40.18, $p<0.001$ for the main effect of color condition; $F(1,14)=20.5, p<0.001$, for the main effect of set size; $F(1,14)=12.15, p=0.003$, for the interaction). Follow-up pairwise comparisons revealed that RTs were significantly faster on trials where the visual search target was encircled in a color that matched the working memory item (target-match) compared to when the memory-matched item encircled a distractor (distractor-match) for set size 1 trials $(t(14)=6.94, p<0.0001, d=1.51)$. A similar pattern of results, albeit with a smaller effect size, was identified when two working memory items were maintained $(t(14)=2.61, p=0.02, d=$ 0.71). A speed-accuracy trade-off is unlikely since participants exhibited roughly equal accuracy on target-match compared to distractor-match search trials (set size 1: $94 \%$ and $92 \%, t(14)=$ 2.07, $p=0.06$; set size $2: 94 \%$ and $93 \%, t(14)=1.63, p=0.13)$.

Despite probing memory on only $20 \%$ of trials, performance estimates were robust and overall quite good (set size 1: TCC $d^{\prime}=3.42$; circular $s d=20.94^{\circ}$; set size 2 : $d^{\prime}=2.56$; swap rate $=0.06$, adjusted $\left.d^{\prime}=2.41, s d=35.05^{\circ}\right)$. Performance was reliably lower at set size 2 relative to set size $1(t(14)=5.78, p<0.001$; see Figure A2). Participants appeared to "swap" and report the non-target memory item nearly $6 \%$ of the time at set size 2 , most likely because the intermediate task induced a loss of location information (mean swap rate for set size 2: 0.06; $t(14)=3.65, p=0.002)$. As noted in the methods, we do not know the strength of memory for the target item on trials where participants misreported the non-target item. Thus, we report d' (memory performance on trials where the correct item was reported; i.e., assuming memory was 
exactly the same strength on swap trials), and adjusted d' (memory performance after adjusting downward to account for swaps, i.e., assuming target memories were non-existent when participants made location swaps). These two estimates provide upper and lower bounds on the true memory strength of target items.

\section{Discussion Experiment A1}

The visual search results of Experiment A1 replicate previous findings showing that working memory items can guide attention towards matching items in a visual search task. Furthermore, as expected, memory performance paralleled the observed search effects, with lower memory performance when two items were held in mind relative to a single item. Since we are averaging performance across trials, both the differences in search effects and the differences in memory performance for 1 vs. 2 items could in principle be driven by overall less efficient processes when two items are held in mind relative to one, or alternatively, could be explained by one strong memory representation which also produced strong guidance on some trials, and one weaker memory representation, which presumably caused less guidance.

\section{Experiment A2: Asymmetric memory strength for multiple working memory items}

In this experiment we examined whether both memory items are represented equally well, or whether memory strength varies between items, such that one item tends to be represented better than the other item. In Experiment A1 we saw that both memory performance and attentional guidance are significantly decreased by holding in mind two items rather than one. This could occur because of averaging across trials and thus averaging over heterogeneity between items at set size 2, or because all items tend to be represented weaker in memory and thus result in smaller guidance effects. 
We adapted the free report technique of Fougnie et al. (2012). Thus, on half of the set size two memory report trials participants were forced to report a randomly probed memory item (50\% chance that either item would be tested, as in Exp. 1) and on the remaining trials, participants were free to choose one of the memory items to report (free report trials). These free report trials allow us to estimate the representational fidelity of a preferred item (preferred simply by nature of being selected) that is presumably the most precise item (Fougnie et al., 2021), and compare it to a randomly probed item.

Similar to Experiment A1, participants were shown either one or two memory items and performed either a visual search or a memory task on every trial, but on half of the set size two memory trials, participants were free to pick one of the memory items to report. On these trials placeholders for both memory items reappeared and participants clicked the location of the item they wished to report. If memory performance on free report trials resembles that of set size one, then we can conclude that variation between items in representational fidelity is large, and consistent with accounts where a single item drives the observed multiple item attentional guidance effect. However, if performance is more similar to set size 2: forced report, then it is less likely that a single item drives the effect and suggests that the reduced effect size for guidance at set size 2 is correlated with the reduced memory precision of the actively maintained working memory items-effectively supporting a multiple-item guidance account.

\section{Methods}

The design, sample size, exclusion criteria, and analysis plan for this experiment were pre-registered using AsPredicted (http://aspredicted.org/blind.php?x=nt3st3).

\section{Participants}

The final sample included 18 participants (10 females) from UC San Diego. Our preregistered exclusion criterion required high visual search performance, and in this case, this 
caused a large number of (thirteen) additional participants to be removed and replaced for failing to achieve $80 \%$ accuracy in the visual search task. In addition, we removed and replaced 3 additional participants for having "swap rates" greater than $40 \%$ of trials, as in Experiment A1. We did not pre-register this swap-based exclusion criterion. However, after analyzing the data, it became clear that these participants failed to follow instructions: such high swap rates indicate that these participants effectively performed "free report" on every trial, regardless of what location was cued, and thus do not provide useful data for distinguishing free vs. forced report memory strength. Although overall this means a high number of participants were excluded (including 13 for the pre-registered criterion and 3 for the swap-based criterion), including all of these participants in the final data set did not alter the results nor their interpretation.

\section{Stimuli}

All aspects of Experiment A2 were identical to Experiment A1 except for free report memory probe trials. On these trials, instead of one test item being cued, two grey circles indicating the possible test items appeared to the left and right of fixation ( $8^{\circ}$ apart) prior to the presentation of the color wheel to allow participants the choice of which item to respond to (which they did by clicking the relevant location).

\section{Procedure}

The following aspects differed in Experiment A2 from Experiment A1. Memory probe trials were evenly split between three conditions: (1) set size one, (2) set size two: forced report, and (3) set size two: free report. All trial types were randomly intermixed throughout the experiment. On free report trials, at test, both items were presented, and participants were instructed to choose one memory item to report, either the left or the right. No further free report instructions were provided (i.e., participants were not incentivized or encouraged to select one item over another). Once a free report selection was made, the color that was encoded at that 
position was set as the correct response and response error was calculated in degrees as the difference between the correct and user selected response.

\section{Model fitting}

We use the TCC model to fit both free report and forced report data. However, the actual response strategy in free report involves not just reporting the color with the strongest familiarity signal (as expected by TCC), but also comparing the two memory items and deciding which item has the stronger memory; something that is not instantiated in the TCC model that is fit to this data. Thus, the d' parameter for the free report fits will not reflect the intrinsic d' that each of the items are represented with but will instead be simply a description of the memory strength that would have been needed for a single item to match the results from the process of choosing the best represented item. The Simulation section following Experiment 3 in the main manuscript addresses this in more detail.
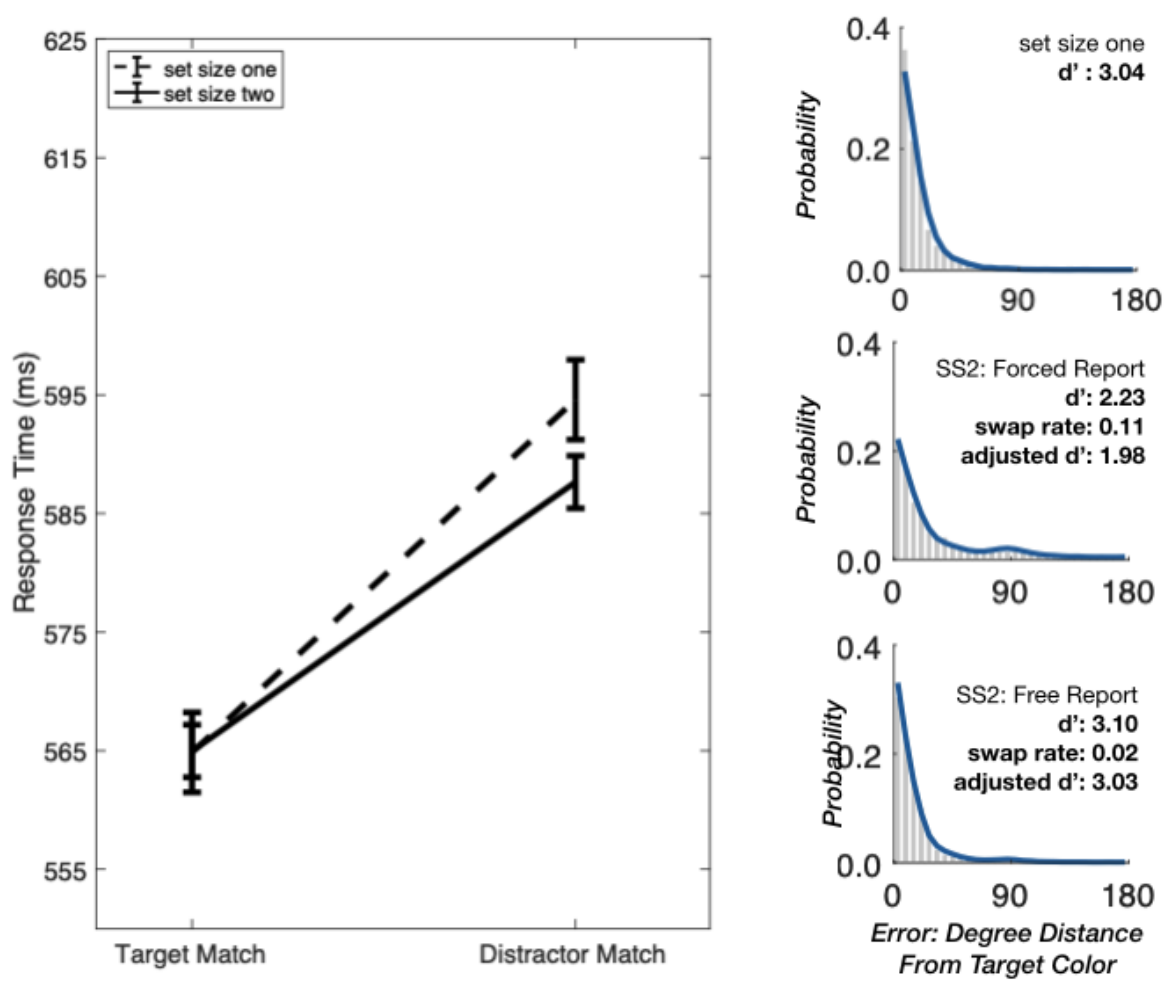
Figure A3. Experiment S2 results. Left: Response times for target and distractor match trials in Experiment A2, separated by working memory set sizes. Replicating Experiment A1, we show robust search benefits for set size 1 and 2. Right: Errors and memory strength from the memory task, visualized with error histograms in gray and model fits in blue. As in Experiment A1, $d$ ' values reflect memory strength on correct-location report trials, and adjusted $d^{\prime \prime}$ values reflect the assumption that participants had no memory for the target on swap trials, giving the range of possible memory strengths depending on assumptions about swaps. Performance on memory trials suggests that at set size 2 , one item ends up with substantially greater representational fidelity compared to the other actively maintained item, as free report performance at set size 2 is as good as set size 1 performance and much better than forced report performance.

\section{Results \& Discussion Experiment A2}

Replicating Experiment $\mathrm{A} 1$, we again found an attentional guidance effect both when one and two items were maintained in working memory. As in Experiment A1, color condition and set size were submitted to a $2 \times 2$ repeated measures ANOVA. This analysis revealed a main effect of color condition $(F(1,17)=28.33, p<0.0001)$ but no main effect of set size $(F(1,17)=0.53, p=0.48)$, nor a reliable interaction $(F(1,17)=2.15, p=0.17)$. Participants were on average faster on target-match trials compared to distractor-match trials for both set size 1 $(t(17)=4.34, p<0.0001)$ and set size $2(t(17)=4.85, p<0.0001)$. Accuracy in this experiment

was again quite good for target-match and distractor-match conditions and there was no evidence of a speed accuracy trade off $(93 \%$ and $91 \%, t(17)=1.46, p=0.16 ; 93 \%$ and $92 \%$ $t(17)=1.34, p=0.20$; for set size 1 and 2 respectively)

With regards to memory performance, we found strong evidence in support of differential representational fidelity between the two items. In particular, when two items were held in working memory, one item was maintained as precisely as if only a single working memory item was remembered (see Figure A3). Thus, memory performance on free-report trials (TCC $d^{\prime}=$ 3.10 , swap rate $=0.02$, adjusted $d^{\prime}=3.03$, circular $s d=27.42^{\circ}$ ) is statistically indistinguishable from performance on set size 1 trials $\left(\mathrm{TCC} d^{\prime}=3.04, s d=27.93^{\circ} ; t(17)=0.62, p=0.55 ; B F_{01}=\right.$ 3.40; Rouder et al., (2009)). Memory performance on random-probe trials (TCC $d^{\prime}=2.23, s d=$ 
$43.78^{\circ}$ ) was comparable to performance from Exp. 1 and while we observed a slightly higher rate of location swap errors here $($ swap rate $=11 \%)$ compared to Exp. $1($ swap rate $=6 \%)$ this difference was not significant $\left(t(31)=1.92, p=0.06 ; B F_{01}=1.33\right)$ suggesting that the inclusion of the Free Report manipulation did not result in a bias to preferentially attend to one compared to both memory items (consistent with validation of this report method from Fougnie et al. 2012).

These results show that, when two working memory items are actively maintained, one item has a stronger memory representation, resulting in considerably more precise color reports at test. This is consistent with accounts where memory items are heterogeneous, either varying in precision intrinsically due to noise that accumulates independently over each item throughout the retention interval (e.g., Fougnie et al. 2012; Schurgin et al. 2020; Wilken \& Ma, 2004), or as a result of a special focus of attention status (Oberauer, 2002; Oberauer \& Lin, 2017).

Furthermore, since one of the two items was maintained with set-size-one-like precision, these results are consistent even with strong versions of these accounts, where the multiple-item guidance effect is a mixture of two kinds of trials: a guidance effect, observed when the search trial contains the high-precision memory item; and a minimal, or non-existent effect, observed when the search trial contains the secondary, low-precision memory item. Notably, however, unlike in Experiment A1, the guidance effect in this experiment was nearly the same size at set size $2(d=1.04)$ as at set size $1(d=1.21)$, which is inconsistent with this "mixture" account. 


\section{References}

Adam, K. C. S., Vogel, E. K., \& Awh, E. (2017). Clear evidence for item limits in visual working memory. Cognitive Psychology, 97, 79-97. https://doi.org/10.1016/j.cogpsych.2017.07.001

Bays, P. M., \& Husain, M. (2008). Dynamic shifts of limited working memory resources in human vision. Science, 321(5890), 851-854. https://doi.org/10.1126/science.1158023

Bays, P. M., Catalao, R. F. G., \& Husain, M. (2009). The precision of visual working memory is set by allocation of a shared resource. Journal of Vision, 9(10), 7-7. https://doi.org/10.1167/9.10.7

Bays, P. M., \& Taylor, R. (2018). A neural model of retrospective attention in visual working memory. Cognitive Psychology, 100(December 2017), 43-52. https://doi.org/10.1016/j.cogpsych.2017.12.001

Beck, V. M., \& Hollingworth, A. (2017). Competition in saccade target selection reveals attentional guidance by simultaneously active working memory representations. Journal of Experimental Psychology: Human Perception and Performance, 43(2), 225-230. https://doi.org/10.1037/xhp0000306

Beck, V. M., Hollingworth, A., \& Luck, S. J. (2012). Simultaneous Control of Attention by Multiple Working Memory Representations. Psychological Science, 23(8), 887-898. https://doi.org/10.1177/0956797612439068

Beck, V. M., \& Vickery, T. J. (2018). Oculomotor capture reveals trial-by-trial neural correlates of attentional guidance by contents of visual working memory. Cortex. https://doi.org/10.1016/j.cortex.2018.09.017

Beck, V. M., \& Vickery, T. J. (2019). Multiple states in visual working memory: Evidence from oculomotor capture by memory-matching distractors.

Brady, T. F., \& Alvarez, G. A. (2015). Contextual effects in visual working memory reveal hierarchically structured memory representations. Journal of Vision, 15(15), 6. 
Memory fidelity drives guidance

https://doi.org/10.1167/15.15.6

Cowan, N. (2000). The magical number 4 in short-term memory: A reconsideration of mental storage capacity Behavioral and Brain Sciences. Behavioral and Brain Sciences, (4), 87185.

Cowan, N. (2005). Working memory capacity. Hove, East Sussex, UK: Psychology Press.

Downing, P. E., \& Dodds, C. M. (2004). Competition in visual working memory for control of search. Visual Cognition, 11(6), 689-703. https://doi.org/10.1080/13506280344000446

Dube, B., \& Al-Aidroos, N. (2019). Distinct prioritization of visual working memory representations for search and for recall. Attention, Perception, and Psychophysics. https://doi.org/10.3758/s13414-018-01664-6

Dube, B., Lumsden, A., \& Al-Aidroos, N. (2019). Probabilistic retro-cues do not determine state in visual working memory. Psychonomic Bulletin and Review, 26(2), 641-646. https://doi.org/10.3758/s13423-018-1533-7

Fan, L., Sun, M., Xu, M., Li, Z., Diao, L., \& Zhang, X. (2019). Multiple representations in visual working memory simultaneously guide attention: The type of memory-matching representation matters. Acta Psychologica, 192, 126-137.

https://doi.org/10.1016/j.actpsy.2018.11.005

Fougnie, D., Suchow, J. W., \& Alvarez, G. A. (2012). Variability in the quality of visual working memory. Nature Communications, 3, 1228-1229. https://doi.org/10.1038/ncomms2237

Frătescu, M., Moorselaar, D. Van, \& Mathot, S. (2018). Can you have multiple attentional templates? Large-scale replications of Van Moorselaar, Theeuwes and Olivers (2014) and Hollingworth and Beck (2016). BioRxiv, III, 474932. https://doi.org/10.1101/474932

Gazzaley, A., \& Nobre, A. C. (2012). Top-down modulation: bridging selective attention and working memory. Trends in Cognitive Sciences, 16(2), 129-135.

https://doi.org/10.1016/j.tics.2011.11.014 
Griffin, I. C., \& Nobre, A. C. (2003). Orienting Attention to Locations in Internal Representations. Journal of Cognitive Neuroscience, 15(8), 1176-1194. https://doi.org/10.1162/089892903322598139

Grubert, A., \& Eimer, M. (2013). Qualitative differences in the guidance of attention during single-color and multiple-color visual search: Behavioral and electrophysiological evidence. Journal of Experimental Psychology: Human Perception and Performance, 39(5), 14331442. https://doi.org/10.1037/a0031046

Hollingworth, A., \& Beck, V. M. (2016). Memory-based attention capture when multiple items are maintained in visual working memory. Journal of Experimental Psychology: Human Perception and Performance, 42(7), 911-917. https://doi.org/10.1037/xhp0000230

Hollingworth, A., \& Hwang, S. (2013). The relationship between visual working memory and attention: Retention of precise colour information in the absence of effects on perceptual selection. Philosophical Transactions of the Royal Society B: Biological Sciences, 368(1628), 20130061. https://doi.org/10.1098/rstb.2013.0061

Hout, M. C., \& Goldinger, S. D. (2014). Target templates: the precision of mental representations affects attentional guidance and decision-making in visual search. Attention, Perception, and Psychophysics, 77(1), 128-149. https://doi.org/10.3758/s13414014-0764-6

Houtkamp, R., \& Roelfsema, P. R. (2006). The effect of items in working memory on the deployment of attention and the eyes during visual search. Journal of Experimental Psychology: Human Perception and Performance, 32(2), 423-442.

\section{https://doi.org/10.1037/0096-1523.32.2.423}

Kerzel, D. (2019). The precision of attentional selection is far worse than the precision of the underlying memory representation. Cognition, 186(January), 20-31. https://doi.org/10.1016/j.cognition.2019.02.001 
Kerzel, D., \& Witzel, C. (2019). The allocation of resources in visual working memory and multiple attentional templates. Journal of Experimental Psychology: Human Perception and Performance, 45(5), 645-658. https://doi.org/10.1037/xhp0000637

Klyszejko, Z., Rahmati, M., \& Curtis, C. E. (2014). Attentional priority determines working memory precision. Vision Research, 105, 70-76.

https://doi.org/10.1016/j.visres.2014.09.002

Lewis-Peacock, J. A., Drysdale, A. T., Oberauer, K., \& Postle, B. R. (2012). Neural evidence for a distinction between short-term memory and the focus of attention. Journal of Cognitive Neuroscience, 24(1), 61-79. https://doi.org/10.1162/jocn a 00140

Macmillan N. A. \& Creelman, C. D. (2005). Detection theory: A user's guide (2nd ed.). Mahwah, NJ: Erlbaum

Mallett, R., \& Lewis-Peacock, J. A. (2018). Behavioral decoding of working memory items inside and outside the focus of attention. Annals of the New York Academy of Sciences, 1424(1), 256-267. https://doi.org/10.1111/nyas.13647

McElree B. (2001). Working memory and focal attention. Journal of experimental psychology. Learning, memory, and cognition, 27(3), 817-835.

Oberauer, K. (2002). Access to Information in Working Memory: Exploring the Focus of Attention. Journal of Experimental Psychology: Learning Memory and Cognition, 28(3), 411-421. https://doi.org/10.1037/0278-7393.28.3.411

Oberauer, K., \& Hein, L. (2012). Attention to Information in Working Memory. Current Directions in Psychological Science, 21(3), 164-169. https://doi.org/10.1177/0963721412444727

Oberauer, K., \& Lin, H. (2017). An Interference Model of Visual Working Memory. Psychological Review, 124(1), 1-39. https://doi.org/10.1037/rev0000044

Olivers, C. N. L. (2009). What Drives Memory-Driven Attentional Capture? The Effects of Memory Type, Display Type, and Search Type. Journal of Experimental Psychology: 
Memory fidelity drives guidance

Human Perception and Performance, 35(5), 1275-1291. https://doi.org/10.1037/a0013896

Olivers, C. N. L., Meijer, F., \& Theeuwes, J. (2006). Feature-based memory-driven attentional capture: Visual working memory content affects visual attention. Journal of Experimental Psychology: Human Perception and Performance, 32(5), 1243-1265.

https://doi.org/10.1037/0096-1523.32.5.1243

Olivers, C. N. L., Peters, J., Houtkamp, R., \& Roelfsema, P. R. (2011). Different states in visual working memory: When it guides attention and when it does not. Trends in Cognitive Sciences, 15(7), 327-334. https://doi.org/10.1016/j.tics.2011.05.004

Panichello, M. F., DePasquale, B., Pillow, J. W., \& Buschman, T. (2018). Error-correcting dynamics in visual working memory. BioRxiv, 319103. https://doi.org/10.1101/319103

Peters, J. C., Goebel, R., \& Roelfsema, P. R. (2009). Remembered but unused: The accessory items in working memory that do not guide attention. Journal of Cognitive Neuroscience, 21(6), 1081-1091. https://doi.org/10.1162/jocn.2009.21083

Pratte, M. S. (2019). Swap errors in spatial working memory are guesses. Psychonomic bulletin \& review, 26(3), 958-966.

Rajsic, J., Ouslis, N. E., Wilson, D. E., \& Pratt, J. (2017). Looking sharp: Becoming a search template boosts precision and stability in visual working memory. Attention, Perception, and Psychophysics, 79(6), 1643-1651. https://doi.org/10.3758/s13414-017-1342-5

Rerko, L., Souza, A. S., \& Oberauer, K. (2014). Retro-cue benefits in working memory without sustained focal attention. Memory and Cognition, 42(5), 712-728.

\section{https://doi.org/10.3758/s13421-013-0392-8}

Robinson, M. M., Benjamin, A. S., \& Irwin, D. E. (2020). Is there a K in capacity? Assessing the structure of visual short-term memory. Cognitive Psychology, 121, 101305.

Rouder, J. N., Speckman, P. L., Sun, D., Morey, R. D., \& Iverson, G. (2009). Bayesian t tests for accepting and rejecting the null hypothesis. Psychonomic Bulletin and Review, 16(2), 225- 
237. https://doi.org/10.3758/PBR.16.2.225

Soto, D., Heinke, D., Humphreys, G. W., \& Blanco, M. J. (2005). Early, involuntary top-down guidance of attention from working memory. Journal of Experimental Psychology: Human Perception and Performance, 31(2), 248-261. https://doi.org/10.1037/0096-1523.31.2.248

Soto, D., Hodsoll, J., Rotshtein, P., \& Humphreys, G. W. (2008). Automatic guidance of attention from working memory. Trends in Cognitive Sciences, 12(9), 342-348. https://doi.org/10.1016/j.tics.2008.05.007

Soto, D., \& Humphreys, G. W. (2007). Automatic Guidance of Visual Attention From Verbal Working Memory. Journal of Experimental Psychology: Human Perception and Performance, 33(3), 730-737. https://doi.org/10.1037/0096-1523.33.3.730

Soto, D., \& Humphreys, G. W. (2008). Stressing the mind: The effect of cognitive load and articulatory suppression on attentional guidance from working memory. Perception and Psychophysics, 70(5), 924-934. https://doi.org/10.3758/PP.70.5.924

Souza, A. S., \& Oberauer, K. (2016). In search of the focus of attention in working memory: 13 years of the retro-cue effect. Attention, Perception, and Psychophysics, 78(7), 18391860. https://doi.org/10.3758/s13414-016-1108-5

Suchow, J. W., Fougnie, D., Brady, T. F., \& Alvarez, G. A. (2014). Terms of the debate on the format and structure of visual memory. Attention, Perception, \& Psychophysics, 76(7), 2071-2079.

Townsend, J. T., \& Wenger, M. J. (2004). The serial-parallel dilemma: A case study in a linkage of theory and method. Psychonomic Bulletin \& Review, 11(3), 391-418.

Van Den Berg, R., Shin, H., Chou, W. C., George, R., \& Ma, W. J. (2012). Variability in encoding precision accounts for visual short-term memory limitations. Proceedings of the National Academy of Sciences of the United States of America, 109(22), 8780-8785. https://doi.org/10.1073/pnas.1117465109 
van Moorselaar, D., Theeuwes, J., \& Olivers, C. N. L. (2014). In competition for the attentional template: Can multiple items within visual working memory guide attention? Journal of Experimental Psychology: Human Perception and Performance, 40(4), 1450-1464. https://doi.org/10.1037/a0036229

Woodman, G. F., \& Luck, S. J. (2007). Do the Contents of Visual Working Memory Automatically Influence Attentional Selection During Visual Search? Journal of Experimental Psychology: Human Perception and Performance, 33(2), 363-377. https://doi.org/10.1037/0096-1523.33.2.363

Zhang, B., Zhang, J. X., Huang, S., Kong, L., \& Wang, S. (2011). Effects of load on the guidance of visual attention from working memory. Vision Research, 51(23-24), 23562361. https://doi.org/10.1016/j.visres.2011.09.008

Zhang, W., \& Luck, S. J. (2008). Discrete fixed-resolution representations in visual working memory. Nature, 453(7192), 233-235. https://doi.org/10.1038/nature06860

Zhou, C., Lorist, M. M., \& Mathôt, S. (2020). Concurrent guidance of attention by multiple working memory items: Behavioral and computational evidence. Attention, Perception, and Psychophysics, 2950-2962. https://doi.org/10.3758/s13414-020-02048-5 